\title{
A vanadium(III) complex with blue and NIR-II spin-flip luminescence in solution
}

Matthias Dorn ${ }^{1}$, Jens Kalmbach², Pit Boden ${ }^{3}$, Ayla Päpcke ${ }^{4}$, Sandra Gómez ${ }^{5}$, Christoph Förster ${ }^{1}$, Felix Kuczelinis ${ }^{1}$, Luca M. Carrella ${ }^{1}$, Laura A. Büldt ${ }^{2}$, Nicolas H. Bings ${ }^{1}$, Eva Rentschler ${ }^{1}$, Stefan Lochbrunner ${ }^{4}$, Leticia González ${ }^{5}$, Markus Gerhards*3, Michael Seitz*2 and Katja Heinze*1

${ }^{1}$ Department of Chemistry, Johannes Gutenberg University of Mainz, Duesbergweg 10-14, 55128 Mainz, Germany.

${ }^{2}$ Institute of Inorganic Chemistry, University of Tübingen, Auf der Morgenstelle 18, 72076 Tübingen, Germany.

${ }^{3}$ Department of Chemistry and Research Center Optimas, TU Kaiserslautern, Erwin-Schrödinger-Straße, 67663 Kaiserslautern, Germany.

${ }^{4}$ Institute for Physics and Department of Life, Light and Matter, University of Rostock, 18051 Rostock, Germany.

${ }^{5}$ Institute of Theoretical Chemistry, Faculty of Chemistry, University of Vienna, Währinger Straße 17, 1090 Vienna, Austria.

\section{Supporting Information}


General Procedures. Diethyl ether and petroleum ether (40/60) were distilled over sodium, acetonitrile over calcium hydride and butyronitrile over $\mathrm{Na}_{2} \mathrm{CO}_{3}$. $\mathrm{VCl}$ (anhydrous, $95 \% ; \mathrm{ABCR}$ ), 10\% $\mathrm{Pd} / \mathrm{C}$ (dry, unreduced; Acros Organics), $\mathrm{CD}_{3} \mathrm{l}$ (>99.5\%D, stabilized with copper, Sigma-Aldrich) and 2,6-diaminopyridine (SigmaAldrich) were used as received. For NMR spectroscopy and synthesis, deuterated solvents with a deuteration level of at least $99.8 \%$ were used. Column chromatography was performed using silica gel 60 (Merck, 0.04$0.063 \mathrm{~mm}$ ). Analytical thin layer chromatography (TLC) was done on silica gel $60 \mathrm{~F} 254$ plates (Merck, coated on aluminum sheets). The ligand $\mathrm{ddpd}^{1}$ and $\left[\mathrm{V}\left(\mathrm{NCCH}_{3}\right)_{6}\right]\left[\mathrm{BPh}_{4}\right]_{2}{ }^{2}$ were prepared according to reported procedures. NMR spectroscopic and mass spectrometric data match the literature values. A glovebox (UniLab/MBraun, Ar 4.8, $\mathrm{O}_{2}<100 \mathrm{ppm}, \mathrm{H}_{2} \mathrm{O}<1 \mathrm{ppm}$ ) was used for storage and weighing of sensitive compounds.

NMR spectra were recorded on a Bruker Avance DRX 400 or AVII+400 NMR spectrometer at $400.31 \mathrm{MHz}$ $\left({ }^{1} \mathrm{H}\right)$. All resonances are reported in ppm versus the solvent signal as internal standard. ${ }^{3}$

IR spectra were recorded with a Bruker Alpha FTIR spectrometer with an ATR unit containing a diamond crystal.

$\mathrm{ESI}^{+}$mass spectra were recorded on a Micromass Q-TOF-Ultima spectrometer by the central analytical facility of the Department of Chemistry (University of Mainz) or on a Bruker Daltonics Esquire 3000 plus mass spectrometer by the central analytical facility of the Department of Chemistry (University of Tübingen).

DC magnetic studies were performed with a Quantum Design MPMS-XL-7 SQUID magnetometer on powdered microcrystalline samples. Experimental susceptibility data were corrected for the underlying diamagnetism using Pascal's constants. The temperature dependent magnetic contribution of the holder and of the embedding eicosane matrix was experimentally determined and subtracted from the measured susceptibility data. Variable temperature susceptibility data were collected in a temperature range of $2-300$ $\mathrm{K}$ under an applied field of 0.1 Tesla.

Electrochemical experiments were carried out on a BioLogic SP-200 voltammetric analyzer using platinum wires as counter and working electrodes and a $0.01 \mathrm{M} \mathrm{Ag} / \mathrm{Ag}\left[\mathrm{NO}_{3}\right]$ electrode as reference electrode. Cyclic voltammetry and square wave measurements were carried out at scan rates of $50-200 \mathrm{mV} \mathrm{s}^{-1} \mathrm{using} 0.1 \mathrm{M}$ $\left[{ }^{\mathrm{n}} \mathrm{Bu}_{4} \mathrm{~N}\right]\left[\mathrm{PF}_{6}\right]$ in $\mathrm{CH}_{3} \mathrm{CN}$ as supporting electrolyte. Potentials are referenced against the ferrocene/ferrocenium couple. UV/Vis/NIR spectra were recorded on a Varian Cary 5000 spectrometer using $1.0 \mathrm{~cm}$ cells.

Luminescence experiments. For luminescence experiments, $\mathrm{CD}_{3} \mathrm{CN}(99.8 \% \mathrm{D}$, NMR grade) or spectrophotometric grade toluene as well as $\mathrm{H}_{2} \mathrm{O}$ were used. Prior to use, all solvents were deoxygenated by three freeze-pump-thaw cycles and eventually sealed under dry, dioxygen-free argon atmosphere. Luminescence measurements at room temperature and at $77 \mathrm{~K}$ were performed on the following instruments:

a) The first instrument was a Horiba Fluorolog-3 spectrofluorimeter equipped with a $450 \mathrm{~W}$ Xenon lamp for steady-state measurements. Emitted light was detected either by a Hamamatsu R2658P PMT detector (200 $\mathrm{nm}<\lambda_{\mathrm{em}}<1010 \mathrm{~nm}$ ) or by a Hamamatsu H10330-75 PMT detector (950 nm $<\lambda_{\mathrm{em}}<1700 \mathrm{~nm}$ ). Spectral selection in the excitation path was accomplished by a DFX monochromator (double gratings: 1200 grooves $/ \mathrm{mm}, 330 \mathrm{~nm}$ blaze) and in the emission paths in the visible/NIR spectral region $\left(\lambda_{\mathrm{em}}<1010 \mathrm{~nm}\right)$ by a spectrograph iHR550 (single gratings: either 1200 grooves $/ \mathrm{mm}, 500 \mathrm{~nm}$ blaze or 950 grooves $/ \mathrm{mm}, 900 \mathrm{~nm}$ blaze) and in the NIR spectral region $\left(\lambda_{\mathrm{em}}>950 \mathrm{~nm}\right.$ ) by a spectrograph iHR320 (single grating: 600 grooves $/ \mathrm{mm}, 1000 \mathrm{~nm}$ blaze). Luminescence lifetimes of the visible emission around $390 \mathrm{~nm}$ was determined at $298 \mathrm{~K}$ in standard fluorescence cuvettes $(1 \mathrm{~cm}$ path length, quartz suprasil) with a pulsed LED (DeltaDiode310, $\lambda_{\mathrm{exc}}=306 \mathrm{~nm} \pm 10 \mathrm{~nm}$, pulse width ca. $1.0 \mathrm{~ns}, P_{\text {avg }}=5 \mu \mathrm{W}$ ). Lifetime data analysis (deconvolution, statistical parameters, etc.) was performed using the software package DAS from Horiba. Lifetimes were 
determined by deconvolution of the decay profiles with the instrument response function, which was determined using a dilute aqueous dispersion of colloidal silica (Ludox ${ }^{\circledR}$ AM-30).

b) The second instrument was a PTI Quantamaster QM4 spectrofluorimeter equipped with a 75 W continuous xenon short arc lamp as excitation source. Emission was monitored using a PTI P1.7R detector module (Hamamatsu PMT R5509-72 with a Hamamatsu C9525 power supply operated at $1500 \mathrm{~V}$ and a Hamamatsu liquid $\mathrm{N}_{2}$ cooling unit $\mathrm{C} 9940$ set to $-80^{\circ} \mathrm{C}$ ). For the measurements between $600 \mathrm{~nm}$ and $900 \mathrm{~nm}$, a long-pass filter GG-455 (Schott, $3.0 \mathrm{~mm}$ thickness, transmission $98-95 \%$ in the spectral region mentioned) was used in the emission channel in order to avoid higher order excitation light. The same approach was taken for NIR measurements above $1000 \mathrm{~nm}$ with a long-pass filter RG-850 (Schott, $3.0 \mathrm{~mm}$ thickness, transmission > $98 \%$ above $970 \mathrm{~nm}$ ). Spectral selection was achieved by single grating monochromators (excitation: 1200 grooves/mm, $300 \mathrm{~nm}$ blaze; vis emission: 1200 grooves $/ \mathrm{mm}, 500 \mathrm{~nm}$ blaze; near-IR emission: 600 grooves/mm, $1200 \mathrm{~nm}$ blaze). Near-IR luminescence lifetimes of the phosphorescent transition ${ }^{1} \mathrm{E}_{\mathrm{g}} /{ }^{1} \mathrm{~T}_{2 \mathrm{~g}} \rightarrow$ ${ }^{3} \mathrm{~T}_{1 \mathrm{~g}}$ were determined at $77 \mathrm{~K}$ (liquid $\mathrm{N}_{2}$ cuvette, sample dissolved in deoxygenated ${ }^{\mathrm{n}} \mathrm{BuCN}$ in standard NMR tubes under argon) with a xenon flash lamp as excitation source (Hamamatsu L4633: pulse width ca. $1.5 \mu \mathrm{s}$ FWHM). Lifetime data analysis (deconvolution, statistical parameters, etc.) was performed using the software package FeliX32 from PTI. Lifetimes were determined by deconvolution of the decay profiles with the instrument response function, which was determined using an empty NMR tube as scatterer.

Partial quantum yields in the visible and the NIR spectral regions were determined using the following equation:

$$
\Phi_{\mathrm{x}}=\Phi_{\mathrm{r}} *\left(\operatorname{Grad}_{\mathrm{x}} / \operatorname{Grad}_{\mathrm{r}}\right) *\left(\eta_{\mathrm{x}}^{2} / \eta_{\mathrm{r}}^{2}\right)
$$

where $\eta$ is the refractive index and Grad is the linearly fitted slope from the plot of the integrated luminescence intensity (corrected for instrument response) versus the absorbance at the excitation wavelength. The subscripts ' $x$ ' and ' $r$ ' refer to the sample and reference, respectively. The vanadium samples were prepared in deoxygenated $\mathrm{CD}_{3} \mathrm{CN}\left(\eta_{\mathrm{x}}=1.341\right)$ at $298 \mathrm{~K}$ under argon (vide supra). For the emission around $390 \mathrm{~nm}$, quinine sulfate in $0.1 \mathrm{M}$ aqueous $\mathrm{H}_{2} \mathrm{SO}_{4}\left(\eta_{\mathrm{r}}=1.33\right)$ was used after excitation at $\lambda_{\text {exc }}=308 \mathrm{~nm}$ as a reference material with a fluorescence quantum yield of $\Phi_{\mathrm{r}}=54.6 \%{ }^{4}$ For the NIR emission band $\left({ }^{1} \mathrm{E}_{\mathrm{g}} /{ }^{1} \mathrm{~T}_{2 \mathrm{~g}}\right.$ $\left.\rightarrow{ }^{3} \mathrm{~T}_{1 \mathrm{~g}}\right), \mathrm{Yb}(\mathrm{tta})_{3}($ phen $)$ in toluene $\left(\eta_{\mathrm{r}}=1.496\right)$ was used after excitation at $\lambda_{\mathrm{exc}}=308 \mathrm{~nm}$ with a quantum yield of $\Phi_{\mathrm{r}}=1.1 \%{ }^{5}$ For the measurement of the ytterbium standard emission, the signal had to be attenuated by a neutral density filter (Newport, neutral density filter FSQ-ND10, transmission around $1000 \mathrm{~nm}$ ca. $11.0 \pm 0.2$ $\%)$ in the emission. The attenuation factor was taken into account mathematically during the integration of the emission bands for the quantum yield calculations.

c) Steady state luminescence measurements in frozen butyronitrile solution down to $20 \mathrm{~K}$ were recorded on a Horiba Jobin Yvon Fluorolog 3-22 $\tau$ spectrometer equipped with a $450 \mathrm{~W}$ xenon lamp and a DSS - IGA020L NIR detector $\left(900 \mathrm{~nm}<\lambda_{\mathrm{em}}<1500 \mathrm{~nm}\right)$. Spectral selection was realized with double and single grating monochromators in the excitation and emission paths, respectively (excitation: 1200 grooves $/ \mathrm{mm}$; near-IR emission 600 grooves/mm). A long-pass filter RG-850 (Edmund Optics, transmission > $98 \%$ above $970 \mathrm{~nm}$ ) was used in the emission channel to avoid higher order excitation light. The butyronitrile solution was cooled down to $20 \mathrm{~K}$ with a closed cycle helium cryostat (ARS Model DE-202A). The cryo cooler was equipped with a cuvette holder and $\mathrm{CaF}_{2}$ windows. Measurements were performed in fluorescence cuvettes $(1 \mathrm{~cm}$ path length, quartz suprasil).

d) Steady state luminescence measurements by using $\mathrm{KBr}$ pellets were performed on a Horiba Jobin Yvon FluoroMax-2 using the described cryostat. A $150 \mathrm{~W}$ xenon lamp was used for sample excitation and detection was realized with a R928 photomultiplier detector. Spectral selection was achieved with single grating monochromators in the excitation and emission paths, respectively (excitation: 1200 grooves $/ \mathrm{mm}, 330 \mathrm{~nm}$ blaze; emission: 1200 grooves $/ \mathrm{mm}, 500 \mathrm{~nm}$ blaze). Long-pass filters with cut-off wavelengths of $420 \mathrm{~nm}$ or 
$600 \mathrm{~nm}$ were used depending on the measurement. $\mathrm{KBr}$ pellets of $\left[\mathrm{V}(\mathrm{ddpd})_{2}\right]\left[\mathrm{PF}_{6}\right]_{3}(\mathrm{ca} .0 .5 \mathrm{mg})$ were prepared by mixing with dry $\mathrm{KBr}$ (ca. $200 \mathrm{mg}$ ), grinding to a homogeneous mixture and pressing into a pellet.

Time-resolved FTIR experiments were performed with an FTIR spectrometer Bruker Vertex 80v, operated in the step-scan mode. $\mathrm{KBr}$ pellets of $\left[\mathrm{V}(\mathrm{ddpd})_{2}\right]\left[\mathrm{PF}_{6}\right]_{3}$ (ca. $0.5 \mathrm{mg}$ ) were prepared by mixing with dry $\mathrm{KBr}$ (ca. $200 \mathrm{mg}$, stored at $80^{\circ} \mathrm{C}$ ) and grinding to a homogeneous mixture. This mixture was filled in an evacuable pellet die with a diameter of $13 \mathrm{~mm}$ and sintered at a pressure of $0.75 \mathrm{GPa}$. The strongest peak in the ground state spectrum showed an absorption of about $0.6 \mathrm{OD}$ with the mentioned concentration. Measurements with cryogenically cooled $\mathrm{KBr}$ pellets ( $20 \mathrm{~K}$ and $290 \mathrm{~K}$ at the sample) were performed with a closed cycle helium cryostat (ARS Model DE-202A). The cryo cooler was equipped with a homebuilt pellet holder and $\mathrm{CaF}_{2}$ windows. A liquid-nitrogen-cooled mercury cadmium telluride (MCT) detector (Kolmar Tech., Model KV1001-B-7/190) with a rise time of $25 \mathrm{~ns}$, connected to a fast preamplifier and a 14-bit transient recorder board (Spectrum Germany, M314142, $400 \mathrm{MS} \mathrm{s}^{-1}$ ), was used for signal detection and processing. The laser setup includes a Q-switched Nd:YAG laser (Innolas SpitLight Evo I) generating pulses with a band-width of $6-9 \mathrm{~ns}$ at a repetition rate of $100 \mathrm{~Hz}$. The third harmonic $(355 \mathrm{~nm})$ of the Nd:YAG laser was used for sample excitation. The UV pump beam was attenuated to about $2.0 \mathrm{~mJ}$ per shot at a diameter of $9 \mathrm{~mm}$. The beam was directed onto the sample and adjusted to have a maximal overlap with the IR beam of the spectrometer. The sample chamber was equipped with anti-reflection-coated germanium filters to prevent the entrance of laser radiation into the detector and interferometer compartments. The time delay between the start of the experiment and the UV laser pulse was controlled with a Stanford Research Systems DG535 delay generator. A total of 10400 coadditions were recorded at each interferogram point. The spectral region was limited by undersampling to $988-1975 \mathrm{~cm}^{-1}$ with a spectral resolution of $4 \mathrm{~cm}^{-1}$ resulting in 555 interferogram points. An IR broad band filter $\left(850-1750 \mathrm{~cm}^{-1}\right.$ ) and the $\mathrm{CaF}_{2}$ windows (no IR transmission $<1000 \mathrm{~cm}^{-1}$ ) of the cryostat prevented problems when performing a Fourier transformation (i.e. no IR intensity outside the measured region should be observed). FTIR ground state spectra were recorded systematically to check for sample degradation. A more detailed description of the step-scan setup is given here. ${ }^{6}$

Transient absorption spectra were recorded applying a pump-probe setup with a time resolution of $100 \mathrm{fs}$. A noncollinear optical parametric amplifier (NOPA) tuned to a center wavelength of $620 \mathrm{~nm}$ was used for excitation and a white light continuum, generated with a $\mathrm{CaF}_{2}$ crystal, for probing. Both the white light generation and the NOPA were pumped by a regenerative Ti:sapphire laser system operating at $775 \mathrm{~nm}$ with a repetition rate of $1 \mathrm{kHz}$. The polarizations of the pump and probe pulses were set to magic angle with respect to each other to avoid effects due to orientational relaxation. Pump and probe beam were focused onto the sample resulting in overlapping spots with diameters of approximately $270 \mu \mathrm{m}$ and $110 \mu \mathrm{m}$, respectively. The sample was dissolved in acetonitrile and filled into a $1 \mathrm{~mm}$ fused silica cuvette. The optical density was set at $600 \mathrm{~nm}$ to $0.3-0.5$ to obtain transient spectra with a good signal to noise ratio. This corresponds to concentrations of $1.8-2.5 \times 10^{-3} \mathrm{M}$ for the complex. The solutions were perfused with argon for approximately two minutes. For the measurements with an excitation wavelength of $400 \mathrm{~nm}$ a second pump-probe setup was used based on a Ti:Sapphire laser system (Spectra-Physics, Spitfire Pro) with a center wavelength of $800 \mathrm{~nm}$ and a repetition rate of $1 \mathrm{kHz}$. By frequency doubling applying a BBO crystal pump pulses at $400 \mathrm{~nm}$ with a pulse duration of $200 \mathrm{fs}$ were obtained. The white light continuum for the probe was again generated with a $\mathrm{CaF}_{2}$ crystal. Both beams with polarizations arranged in magic angle were focused onto the sample leading to spots with diameters of $170 \mu \mathrm{m}$ for the pump and $80 \mu \mathrm{m}$ for the probe. The sample was dissolved in $\mathrm{CH}_{3} \mathrm{CN}$ and filled into a $1 \mathrm{~mm}$ fused silica cuvette. The optical density was set to 0.7 at $400 \mathrm{~nm}$ which corresponds to an optical density of 0.4 at $600 \mathrm{~nm}$ and to a concentration of $2.5 \times 10^{-3} \mathrm{M}$. The same sample was used as in the aforementioned measurement with an excitation at $620 \mathrm{~nm}$. 
Elemental analyses were conducted by the microanalytical laboratory of the department of chemistry of the University of Mainz.

Trace determination of chromium in the presence of vanadium. Mass spectrometrical analysis of the starting materials for traces of $\mathrm{Cr}$ were performed by the Laboratory for Inorganic Trace Analysis and Plasma Spectrometry, University of Mainz. The chromium concentrations in $\mathrm{VCl}_{3}(\mathrm{THF})_{3}$, and and $\mathrm{VCl}_{3}\left(\mathrm{CH}_{3} \mathrm{CN}\right)_{3}$ were determined by microwave assisted acid digestion and subsequent SN-ICP-MS (solution nebulizationinductively coupled plasma-mass spectrometry) measurement. Initially, the approximate chromium concentrations were determined via digestion and subsequent ICP-MS measurement of $\sim 10 \mathrm{mg}$ of the respective compound using a three point external calibration with concentrations between $0.5 \mathrm{ng} \mathrm{ml}^{-1}$ and $50 \mathrm{ng} \mathrm{ml}^{-1}$. Based on these results, the procedure to achieve valid quantitative results was as follows: An amount of (40-130) mg per sample was weighted and digested in $6 \mathrm{~mL}$ nitric acid (70\% p.a. grade, Fisher Chemical, Fisher Scientific $\mathrm{GmbH}$, Germany) and $2 \mathrm{ml}$ hydrogen peroxide solution (30\% p.a. grade, ROTIPURAN $^{\circledR}$, Carl Roth, Germany) using an Ethos 2000plus microwave assisted digestion system (Milestone Inc, Shelton, CT, USA). The first step of the heating program was a temperature ramp to $140{ }^{\circ} \mathrm{C}$ within 7.5 min followed by a second ramp to $200{ }^{\circ} \mathrm{C}$ within $5 \mathrm{~min}$. This temperature was held for $10 \mathrm{~min}$ and afterwards increased to $220^{\circ} \mathrm{C}$ within $5 \mathrm{~min}$ before holding this temperature for $20 \mathrm{mins}$. Prior to digestion, $100 \mu \mathrm{l}$ of a $10 \mu \mathrm{g} \mathrm{ml}^{-1} Y$-solution were added to compensate for analyte loss during the digestion and subsequent sample preparation steps. The obtained solutions were diluted to $25 \mathrm{ml}$ with ultrapure water (Millipore, Milli-Q System, USA) from which five aliquots of $1 \mathrm{ml}$ were diluted to $10 \mathrm{ml}$ each. For quantification via standard addition, $\mathrm{Cr}$ and $\mathrm{Y}$ were added at concentrations between 0 and $5 \mathrm{ng} \mathrm{ml}^{-1}$, and 0 and $8 \mathrm{ng} \mathrm{ml}^{-1}$, respectively. Sc was added at a concentration of $4 \mathrm{ng} \mathrm{ml}^{-1}$ as internal standard. For ICP-MS measurements, a sample introduction system consisting of a concentric MicroMist ${ }^{\mathrm{TM}}$ nebulizer (Agilent Technologies, Santa Clara, CA, USA) and a double pass spray chamber (Agilent Technologies, Santa Clara, CA, USA) were coupled to a quadrupole ICP-MS instrument 7800 (Agilent Technologies, Santa Clara, CA, USA). The signal intensities of ${ }^{45} \mathrm{SC}^{+},{ }^{52} \mathrm{Cr}^{+},{ }^{53} \mathrm{Cr}^{+}$and ${ }^{89} \mathrm{Y}^{+}$were measured for data evaluation. The optimum instrumental parameters of the

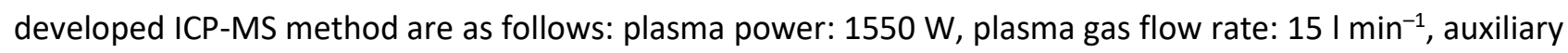
gas flow rate: $0.9 \mathrm{I} \mathrm{min}^{-1}$, nebulizer gas flow rate: $0.99-1.05 \mathrm{I} \mathrm{min}^{-1}$, sampler and skimmer cone: $\mathrm{Ni}$, He gas flow rate (cell gas): $2 \mathrm{ml} \mathrm{min}^{-1}$, data acquisition: 0.3 s integration time, 30 sweeps per replicate, 6 replicates.

$\mathrm{VCl}_{3}(\mathrm{NCMe})_{3}:(4.01 \pm 0.31) \mu \mathrm{g} / \mathrm{g}(U ; k=2) ; \mathrm{VCl}_{3}(\mathrm{THF})_{3}:(3.45 \pm 0.10) \mu \mathrm{g} / \mathrm{g}(U ; k=2)$.

Crystal structure determination. Intensity data were collected with a STOE IPDS-2T diffractometer and an Oxford cooling system and corrected for absorption and other effects using Mo $K_{\alpha}$ radiation $(\lambda=0.71073 \AA$ ). The diffraction frames were integrated using the STOE X-Area software package ${ }^{7}$, and most were corrected for absorption with MULABS ${ }^{8}$ of the PLATON package. ${ }^{9}$ The structure was solved by direct methods and refined by the full-matrix method based on $F^{2}$ using the SHELXTL software package. ${ }^{10,11}$ All non-hydrogen atoms were refined anisotropically, while the positions of all hydrogen atoms were generated with appropriate geometric constraints and allowed to ride on their respective parent carbon atoms with fixed isotropic thermal parameters. CCDC $1958562\left(\left[\mathrm{~V}(\mathrm{ddpd})_{2}\right]\left[\mathrm{PF}_{6}\right]_{3} \times 3 \mathrm{CH}_{3} \mathrm{CN}\right)$ contains the supplementary crystallographic data for this paper. These data are provided free of charge by The Cambridge Crystallographic Data Centre.

Crystallographic Data of mer-[V(ddpd) $\left.)_{2}\right]\left[\mathrm{PF}_{6}\right]_{3} \times 3 \mathrm{CH}_{3} \mathrm{CN}$. $\mathrm{C}_{40} \mathrm{H}_{43} \mathrm{~F}_{18} \mathrm{~N}_{13} \mathrm{P}_{3} \mathrm{~V}(1191.72)$; triclinic; $P \overline{1} ; a=11.483(2)$ $\AA, b=11.901(2) \AA, c=18.093(4) \AA, \alpha=90.88(3)^{\circ}, \beta=96.38(3)^{\circ}, \gamma=91.60(3)^{\circ} ; V=2456.0(9) \AA^{3} ; Z=2$; density, calcd. $=1.612 \mathrm{~g} \mathrm{~cm}^{-3}, T=193(2) \mathrm{K}, \mu=0.415 \mathrm{~mm}^{-1} ; \mathrm{F}(000)=1208 ;$ crystal size $0.620 \times 0.520 \times 0.460 \mathrm{~mm} ; \theta=$ 2.437 to 28.426 deg.; $-15 \leq \mathrm{h} \leq 15,-15 \leq \mathrm{k} \leq 14,-24 \leq \mathrm{I} \leq 24$; rfln collected $=25922$; rfln unique $=12253$ [ $R$ (int) = 0.0416]; completeness to $\theta=25.242 \mathrm{deg}$. $=99.8 \%$; semi empirical absorption correction from equivalents; max. and min. transmission 1.11737 and 0.90264 ; data 12253; restraints 84, parameters 747; goodness-offit on $F^{2}=1.028$; final indices $[I>2 \sigma(I)] R_{1}=0.0409, \mathrm{w} R_{2}=0.1060 ; R$ indices (all data) $R_{1}=0.0548, \mathrm{w} R_{2}=$ 0.11126; largest diff. peak and hole 0.391 and -0.489 e $\AA^{-3}$. 


\section{Computational Details.}

The characterization of the absorption spectrum was done employing two types of quantum chemical calculations: i) density functional theory in its unrestricted form, as for open shell species unrestricted orbitals are expected to deliver best geometries and energy values in comparison to restricted orbitals, and ii) multiconfigurational theory with an active space tailored to predict accurately the MC states. The first method is labelled below as "Unrestricted Kohn-Sham orbitals DFT (UKS), the second as "SOC-CASSCF(6,12)FIC-NEVPT2". These two methods are complementary to each other, as the first gives the best energetics for the LMCT states, while the second provides accurately the position of the MC states. The simulation of the excited state molecular dynamics states related to the spin-flip was done using surface hopping, below labeled as "non-adiabatic spin-flip dynamics calculations". As explained in more detailed here, the electronic structure theory calculations underlying the molecular dynamics were done with the same computational protocol as the DFT calculations, but using restricted orbitals, in order to obtain pure spin states.

Static unrestricted Kohn-Sham orbitals DFT (UKS): All calculations were performed using the quantum computing suite ORCA 4.0.1. ${ }^{12}$ Geometry optimization (Tables S1 - S2) was performed using unrestricted Kohn-Sham orbitals DFT (UKS) and the B3LYP functional ${ }^{13}$ in combination with Ahlrichs' split-valence triple- $\zeta$ basis set def2-TZVPP for all atoms. ${ }^{14,15}$ Tight convergence criteria were chosen for DFT-UKS calculations (keywords tightscf and tightopt). All DFT-UKS calculations make use of the resolution of identity (Split-RI-J) approach for the Coulomb term in combination with the chain-of-spheres approximation for the exchange term (COSX). ${ }^{16,17}$ The zero order relativistic approximation was used to describe relativistic effects in all calculations (keyword ZORA). ${ }^{18,19}$ Grimme's empirical dispersion correction D3(BJ) was employed (keyword D3BJ). ${ }^{20,21}$ To account for solvent effects, a conductor-like screening model (keyword CPCM) modeling acetonitrile was used in all calculations. ${ }^{22}$ Explicit counterions and/or solvent molecules were neglected. TDDFT-UKS calculations were performed at the same level of theory using unrestricted Kohn-Sham orbitals (UKS). Fifty vertical spin-allowed transitions were calculated (Tables S3 - S4).

SOC-CASSCF(6,12)-FIC-NEVPT2: Calculations of ground- and excited-state properties with respect to metalcentered $(\mathrm{MC})$ states were performed using the complete-active-space self-consistent field method (including spin-orbit coupling (SOC-CASSCF) ${ }^{23,24}$ for calculation of the zero-field splitting $D$ ) in conjunction with the fully internally contracted N-electron valence perturbation theory to second order (FIC-NEVPT2) ${ }^{25,26}$ in order to recover missing dynamic electron correlation. SOC was treated through the mean-field (SOMF) approximation ${ }^{27,28}$, and the effective Hamiltonian approach ${ }^{29-31}$ was used to compute the spin-Hamiltonian parameters. In order to accurately model the ligandfield, active spaces were chosen to encompass the dominate bonding/antibonding orbitals formed between vanadium and the ligand. An active space of $(6,12)$ along with 10 triplet roots and 12 singlet roots was selected (Tables S5 - S6). In addition to the minimal active space of $(2,5)$, two occupied $\mathrm{V}-\mathrm{N} \sigma$ bonding orbitals and a second $\mathrm{d}$ shell ${ }^{32}$ were included in these calculations (Table S5).

Non-adiabatic spin-flip dynamic calculations were accomplished using the SHARC (Surface Hopping including ARbitrary Couplings) ${ }^{33-36}$ molecular dynamics program suite with the pySHARC driver. The electronic Hamiltonian was approximated using a linear vibronic coupling (LVC) method ${ }^{37}$ based on TDDFT vertical excitations calculated using the methodological details described above with one essential difference. The open-shell (UKS) ground state electronic wave function used as a reference for the previous TDDFT calculations delivers electronic states that cannot be labelled according to their spin multiplicity (i.e., they are not eigenfunctions of the spin operator). As we need explicit couplings between pure singlet and pure triplet states, a RKS approach, where the two unpaired electrons are forced to be paired in one restricted orbital, is used to do single point calculations on the UKS optimized geometry and obtain pure triplet states as a linear response of a pure singlet state. The obtained RKS energies are also listed in Table S3. Since there is only one reference closed-shell orbital instead of two - as one would have in an open-shell triplet state some electronic states are missing in the RKS approach with respect to the UKS calculation. In the RKS 
approach, the assignment of the state characters has been done dividing the molecule into three fragments (metal center and two ddpd ligands) and calculating charge transfer (CT) numbers, as implemented in the TheoDore software package. ${ }^{38}$ The quantitative wave function analysis is presented in Fig. S21 and the largest contribution is used to state the main state character in Table S3.

A total of 48 coupled diabatic electronic states (12x3 triplets and 12 singlets, listed in Table S8) were included in the SHARC-LVC calculations. For the $100 \mathrm{fs}$ simulations (Fig. S22), 4 sets of 54, 69, 60, 59 trajectories were excited $100 \%$ to the lowest $1^{3} \mathrm{LMCT}, 2^{3} \mathrm{LMCT}, 3^{3} \mathrm{LMCT}$ and $4^{3} \mathrm{LMCT}$ states, respectively. In the 50 ps simulation (Figure 4), 294 trajectories were included, starting from the $2^{3}$ LMCT state, which was identified as the longest-lived state during the previous $100 \mathrm{fs}$. The nuclear motion was propagated with a $0.5 \mathrm{fs}$ step, and the electronic wave function was integrated with a $0.02 \mathrm{fs}$ step, using the local diabatization scheme. ${ }^{39}$ During a hop, the full momentum vector was rescaled to conserve total energy. The energy-based decoherence correction ${ }^{40}$ with the standard parameter of 0.1 a.u. was used. 1000 initial geometries were sampled from a harmonic Wigner distribution of the UKS ground state; from the 231 available normal modes, one imaginary mode $\left(-30 \mathrm{~cm}^{-1}\right.$ ) and the highest 33 (from 3047 to $3279 \mathrm{~cm}^{-1}$ ) were excluded due to anharmonicities. These modes correspond to the large amplitude torsions of the methyl groups, which cannot be described by a linear model potential. For each of the remaining 196 normal modes, 4415 intrastate and 18827 interstate vibronic couplings were calculated using a numerical differentiation scheme and the computation of wave function overlaps between the states at the reference geometry and the displaced geometries. We note that the optimized geometry of the singlet reference state and that of the triplet ground state are very similar $($ RMSD $=0.584)$, justifying the use of the UKS frequencies as seed for the initial conditions.

Using the LVC framework, the optimization of the longest-lived singlet state $\left(2^{1} \mathrm{MC}\right)$ leads to the structure given in Table S9. This state lays $1.1 \mathrm{eV}$ above the triplet ground state and it is assigned as the NIR emissive state. 


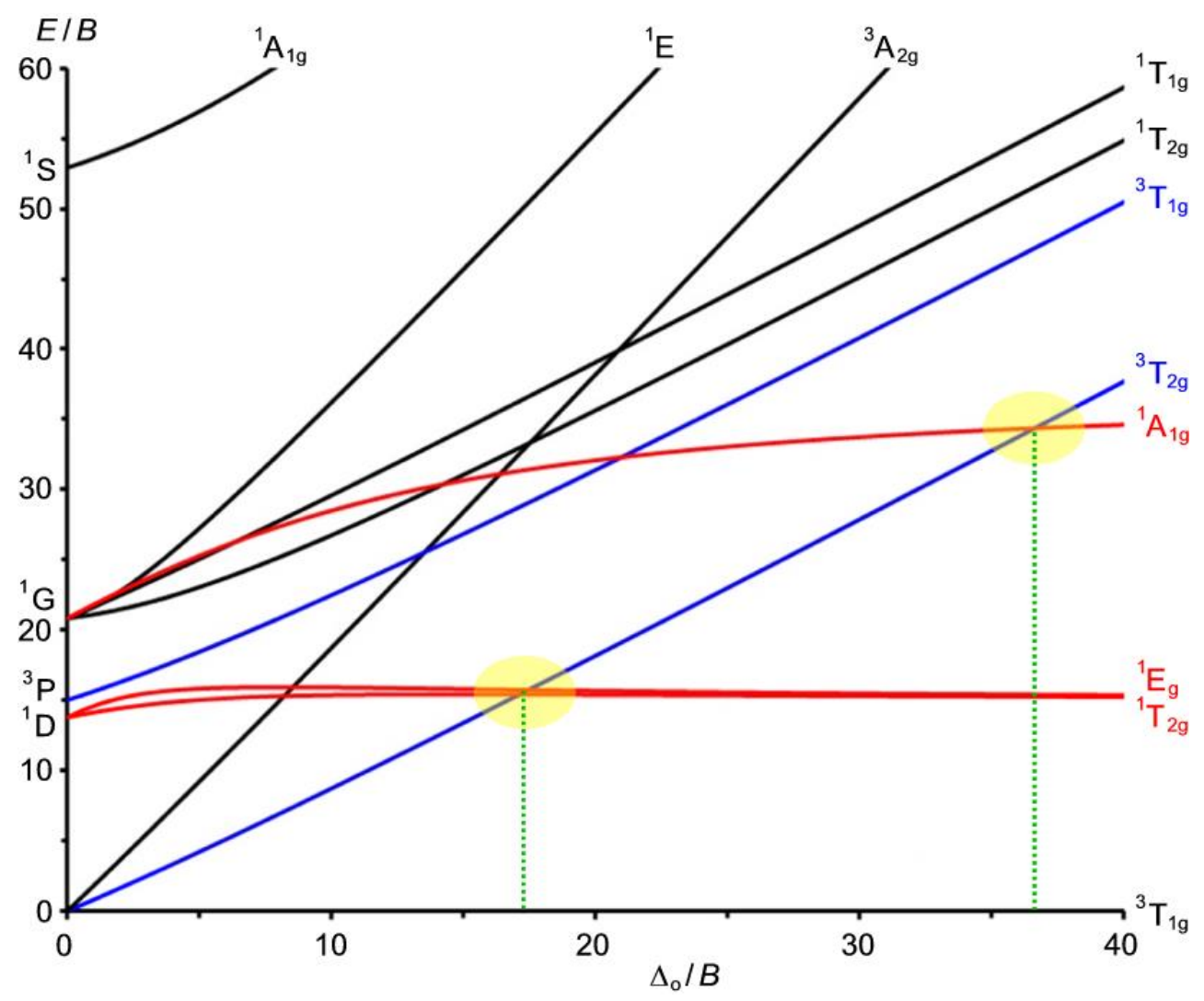

Figure S1. Tanabe Sugano diagram for octahedral transition metal complexes with $\mathrm{d}^{2}$ electron configuration $(C / B=4.42)$. Relevant excited triplet states in blue, relevant singlet states in red, important crossing points highlighted in yellow. ${ }^{42}$

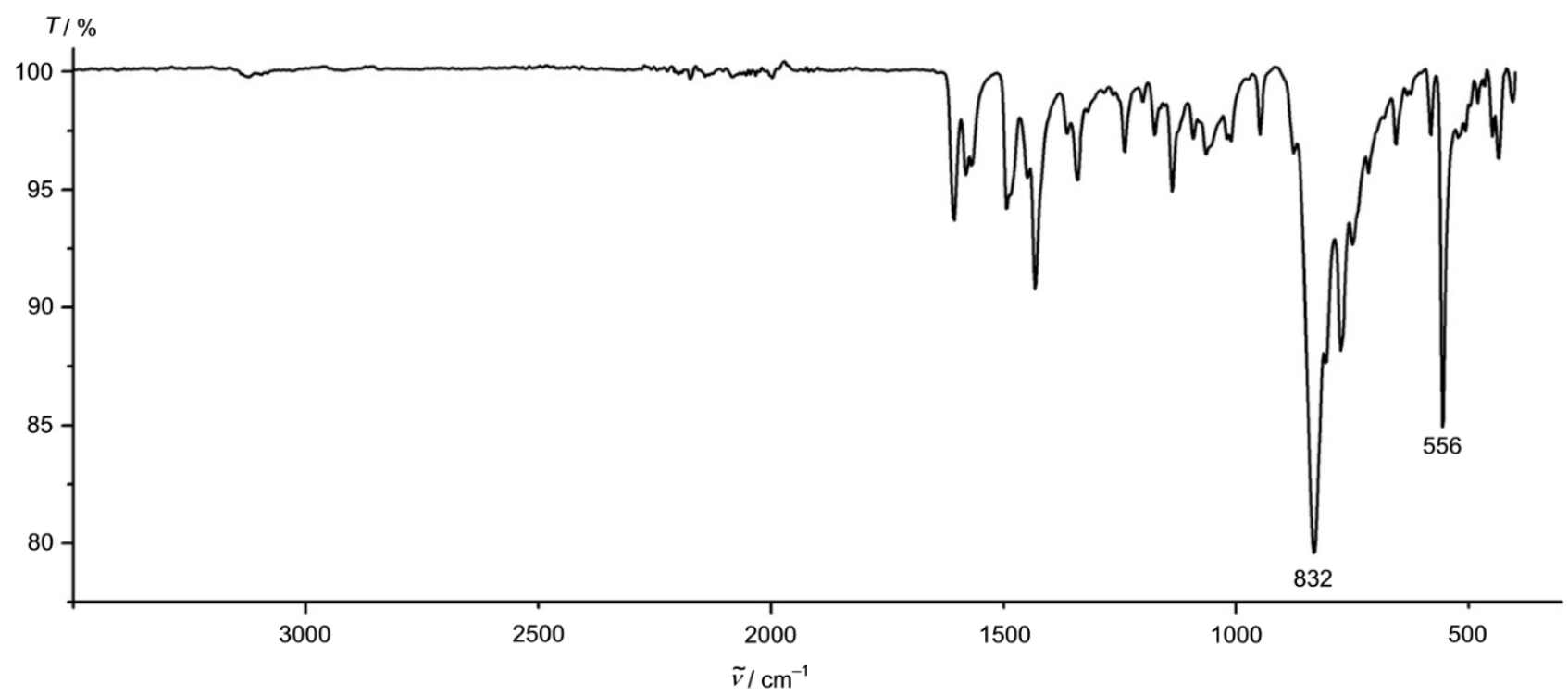

Figure S2. ATR IR spectrum of $m e r-\left[V^{\prime \prime \prime}(d d p d)_{2}\right]\left[\mathrm{PF}_{6}\right]_{3}$. 


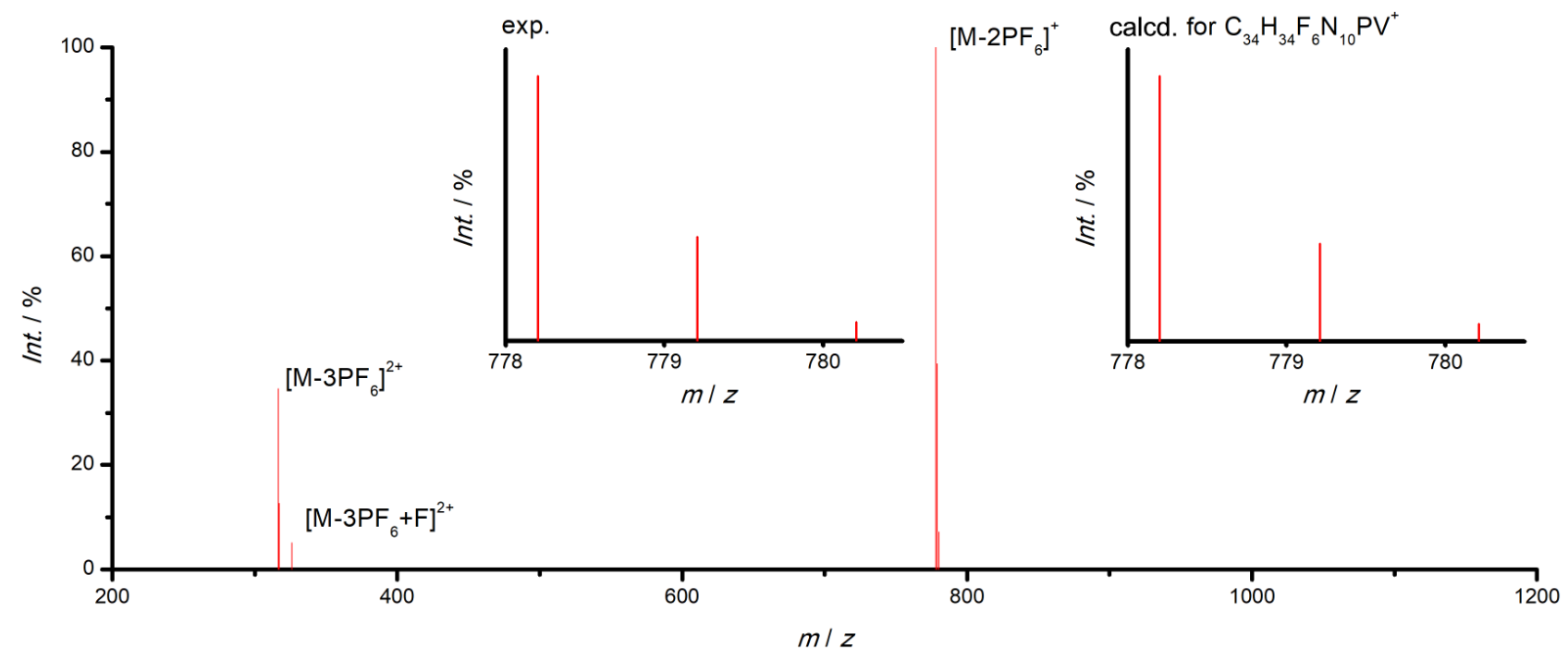

Figure S3. $\mathrm{ESI}^{+}$mass spectrum of mer-[V'II'(ddpd $\left.)_{2}\right]\left[\mathrm{PF}_{6}\right]_{3}$ in $\mathrm{CH}_{3} \mathrm{CN}$. The insets show the experimental and calculated isotopic pattern of the largest peak.

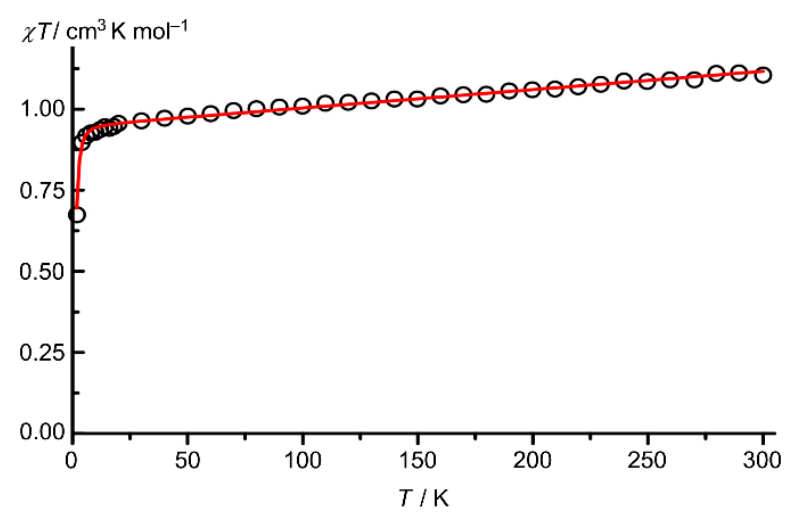

Figure S4. Temperature-dependence of $\chi_{\mathrm{M}} T$ of mer-[V(ddpd $\left.)_{2}\right]\left[\mathrm{PF}_{6}\right]_{3}$ (black circles) with fit (red line, $g=$ 1.946(2), $D=+2.87(11) \mathrm{cm}^{-1} ; \mathrm{TIP}=566 \times 10^{-6} \mathrm{~cm}^{3} \mathrm{~K} \mathrm{~mol}^{-1}$ ). Simulation with a negative $D$ value gave a slightly poorer fit to the experimental data. As obtained by HF-EPR spectroscopy, reported vanadium(III) complexes exhibit positive $D$ values. ${ }^{43}$ Only two oxalates of vanadium(III) are reported to possess negative $D$ values based on inelastic neutron scattering and single crystal high-resolution FT absorption spectroscopy. ${ }^{44}$

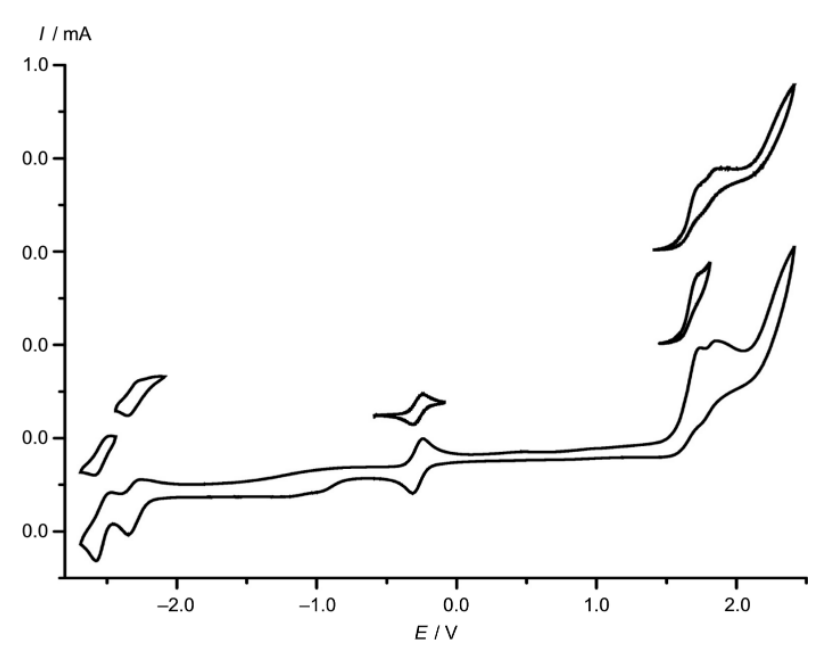

Figure S5. Cyclic voltammogram of mer-[V'I'(ddpd $\left.)_{2}\right]\left[\mathrm{PF}_{6}\right]_{3}$ in $\left[{ }^{\mathrm{n}} \mathrm{Bu}_{4} \mathrm{~N}\right]\left[\mathrm{PF}_{6}\right]$ in $\mathrm{CH}_{3} \mathrm{CN} ; E$ vs. ferrocene. 


\section{Synthesis and characterization of $\left[D_{17}\right]-d d p d$}

$\left[D_{4}\right]-2-C h l o r o p y r i d i n e^{45}$

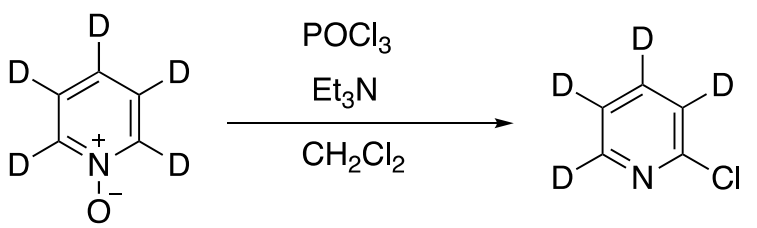

To a stirred solution of [ $\mathrm{D}_{5}$ ]-pyridine- $\mathrm{N}$-oxide ${ }^{46}(8.1 \mathrm{~g}, 81 \mathrm{mmol}, 1.0$ eq.) and triethylamine $(13.4 \mathrm{ml}, 9.8 \mathrm{~g}, 97$ mmol, 1.2 eq.) in dichlormethane $(80 \mathrm{ml})$ was slowly added a solution of $\mathrm{POCl}_{3}(8.8 \mathrm{ml}, 14.9 \mathrm{~g}, 97 \mathrm{mmol}, 1.2$ eq.) in dichlormethane $(50 \mathrm{ml})$ at $10{ }^{\circ} \mathrm{C}$. After stirring for 30 minutes at room temperature, the reaction mixture was refluxed for $1 \mathrm{~h}$. The solution was cooled to room temperature and ice was slowly added until all excess phosphoroxychloride was hydrolysed. After addition of water $(30 \mathrm{ml})$, the solution $\mathrm{pH}$ was adjusted to 10 with aq. $\mathrm{NaOH}(2 \mathrm{M})$. The organic layer was separated and the aqueous layer was extracted with $\mathrm{CH}_{2} \mathrm{Cl}_{2}$ $(5 \times 30 \mathrm{ml})$. The combined organic layers were washed with a saturated $\mathrm{NaCl}$ solution, dried $\left(\mathrm{MgSO}_{4}\right)$ and concentrated at reduced pressure. The resulting red oil was subjected to column chromatography $\left(\mathrm{SiO}_{2}\right.$, $\mathrm{CH}_{2} \mathrm{Cl}_{2}$, preloading onto $\mathrm{SiO}_{2}$, detection: UV). The product was obtained as a colorless oil $(3.8 \mathrm{~g}, 40 \%,>99.5$ $\% \mathrm{D})$.

${ }^{13} \mathrm{C}\left\{{ }^{1} \mathrm{H}\right\} \mathrm{NMR}\left(101 \mathrm{MHz}, \mathrm{CDCl}_{3}\right): \delta=151.4(\mathrm{~s}), 149.3(\mathrm{t}, J=28.0 \mathrm{~Hz}), 138.3(\mathrm{t}, J=25.0 \mathrm{~Hz}), 124.0(\mathrm{t}, J=26.2 \mathrm{~Hz})$, $121.7(\mathrm{t}, J=25.6 \mathrm{~Hz})$ ppm. MS $\left(\mathrm{ESI}^{+}\right): \mathrm{m} / z(\%)=118.04\left(100,[\mathrm{M}+\mathrm{H}]^{+}\right)$.

\section{$\left[D_{3}\right]$-2,6-Diaminopyridine}

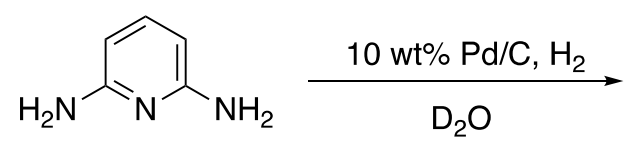<smiles>[2H]c1c(N)nc(N)c([2H])c1[2H]</smiles>

In analogy to a published procedure, ${ }^{47}$ a mixture of 2,6-diaminopyridine (500 mg, $4.6 \mathrm{mmol}, 1.0 \mathrm{eq}$.) and 10 $\% \mathrm{Pd} / \mathrm{C}(10 \mathrm{wt}-\%, 50 \mathrm{mg})$ in $\mathrm{D}_{2} \mathrm{O}(20 \mathrm{ml}$, NMR grade, $99.9 \% \mathrm{D})$ in a steel autoclave was set under an atmosphere of $\mathrm{H}_{2}$ (1 bar) and stirred at $180^{\circ} \mathrm{C}$ (bath temperature) for $24 \mathrm{~h}$. After cooling to room temperature, methanol $(20 \mathrm{ml})$ was added and the dark green reaction mixture was filtered through celite. The filter cake was washed with additional methanol $(3 \times 10 \mathrm{ml})$ and the combined filtrate was concentrated under reduced pressure. The product was obtained as dark green solid $\left(510 \mathrm{mg}, 99\right.$ \%, $\mathrm{H}^{3 / 5}: 87$ \% , H4: 97 \% , overall: 90.5 $\% \mathrm{D})$.

${ }^{1} \mathrm{H}$ NMR (400 MHz, CD $\left.30 D\right): \delta=7.18(0.03 \mathrm{H}$, non-deuterated residual signal), $5.84(0.25 \mathrm{H}$, non-deuterated residual signal), $4.84(\mathrm{~s}, 4 \mathrm{H}) \mathrm{ppm}$. MS $\left.(\mathrm{ESI})^{+}\right): m / z(\%)=113.1\left(100,[\mathrm{M}+\mathrm{H}]^{+}\right)$.

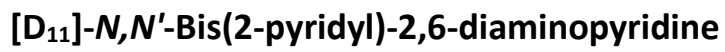<smiles>[2H]c1c(N)nc(N)c([2H])c1[2H]</smiles><smiles>[2H]c1nc(Cl)c([2H])c([2H])c1[2H]</smiles>

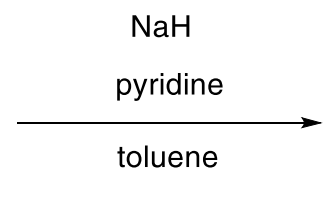<smiles>[2H]c1nc(Nc2nc(Nc3nc([2H])c([2H])c([2H])c3[2H])c([2H])c([2H])c2[2H])c([2H])c([2H])c1[2H]</smiles>

In analogy to a published procedure ${ }^{48}$ under an argon atmosphere a mixture of $\left[D_{3}\right]$-2,6-diaminopyridine $(0.5$ g, $4.4 \mathrm{mmol}, 1$ eq.) and $\mathrm{NaH}$ (60 wt\% dispersion in mineral oil, $1.2 \mathrm{~g}, 29 \mathrm{mmol}, 6.8$ eq.) was stirred for $5 \mathrm{~min}$ 
at room temperature. [ $\left.\mathrm{D}_{4}\right]$-2-Chloropyridine $(1.2 \mathrm{ml}, 1.5 \mathrm{~g}, 13 \mathrm{mmol}, 3$ eq. $)$ and pyridine $(2.5 \mathrm{ml})$ were added, followed by stirring for $5 \mathrm{~min}$ at room temperature. The reaction mixture was immersed in a preheated oil bath at $150{ }^{\circ} \mathrm{C}$ resulting in immediate, strong gas evolution. A few minutes later, just before the red brown suspension became a solid mass, dry toluene $\left(15 \mathrm{ml}\right.$ ) was added. The mixture was stirred at $150^{\circ} \mathrm{C}$ (bath temperature) for $4 \mathrm{~h}$. After cooling to room temperature, the solvent was removed under reduced pressure. Cautiously, ice-cold water $(30 \mathrm{ml})$ was added dropwise. After stirring for 10 minutes, the brown precipitate was collected on a sintered-glass frit (porosity 3 ), washed extensively with water $(3 \times 20 \mathrm{ml}$ ) and $n$-hexane (3 $\times 10 \mathrm{ml})$. The light brown solid was dissolved in THF $(60 \mathrm{ml})$ and filtered through a short plug of silica. The plug was washed with additional THF $(3 \times 10 \mathrm{ml})$ and the combined THF filtrates were concentrated under reduced pressure resulting in a brown solid. The crude product was transferred into a $25 \mathrm{ml}$ round bottom flask and $\mathrm{MeOH}(3 \mathrm{ml}), \mathrm{CH}_{2} \mathrm{Cl}_{2}(3 \mathrm{ml})$ and $\mathrm{Et}_{2} \mathrm{O}(3 \mathrm{ml})$ were added. After stirring for $3 \mathrm{~h}$ at room temperature, the mixture was cooled in an ice bath. The beige solid was collected on a filter, washed with $\mathrm{Et}_{2} \mathrm{O}(3 \times 5 \mathrm{ml})$ and dried under vacuum. The product was obtained as beige solid (0.36 g, 29\%, 90.3\%D).

${ }^{1} \mathrm{H}$ NMR (400 MHz, [ $\left.\left.\mathrm{D}_{6}\right]-\mathrm{DMSO}\right): \delta=9.36(\mathrm{~s}, 2 \mathrm{H}), 7.12(0.9 \mathrm{H}$, non-deuterated residual signal) ppm.

\section{$\left[D_{17}\right]-$ ddpd $^{49}$}<smiles>[2H]c1nc(Nc2nc([2H])c([2H])c([2H])c2Nc2nc([2H])c([2H])c([2H])c2[2H])c([2H])c([2H])c1[2H]</smiles><smiles></smiles>

Under an argon atmosphere, powdered $\mathrm{KOH}$ (99 mg, $1.8 \mathrm{mmol}, 4.0$ eq.) was added to a yellow solution of [ $\left.D_{11}\right]-N, N^{\prime}$-bis(2-pyridyl)-2,6-diaminopyridine $\left(120 \mathrm{mg}, 0.4 \mathrm{mmol}, 1.0\right.$ eq.) in [ $\mathrm{D}_{6}$ ]-DMSO (10 ml, NMR grade, 99.8 \%D). The mixture was stirred at room temperature for $30 \mathrm{~min} . \mathrm{CD}_{3} \mathrm{l}(60 \mu \mathrm{l}, 140 \mathrm{mg}, 0.97 \mathrm{mmol}, 2.2 \mathrm{eq}$.) was added to the red reaction solution and stirring was continued overnight $(16 \mathrm{~h})$. Water $(40 \mathrm{ml})$ was added and the yellow aqueous suspension was extracted with a $1: 1(\mathrm{v} / \mathrm{v})$ mixture of $\mathrm{Et}_{2} \mathrm{O} / \mathrm{THF}(3 \times 30 \mathrm{ml})$. The combined organic phases were washed with aqueous sat. $\mathrm{Na}_{2} \mathrm{CO}_{3}(25 \mathrm{ml})$, dried $\left(\mathrm{MgSO}_{4}\right)$ and concentrated under reduced pressure. The resulting yellow oil was subjected to column chromatography $\left(\mathrm{SiO}_{2}, \mathrm{n}\right.$ hexane/EtOAc, 12:1) to obtain the product as a pale-yellow oil (75 mg, $55 \%,>93.6 \% \mathrm{D}$ ).

${ }^{1} \mathrm{H} \mathrm{NMR}\left(400 \mathrm{MHz}, \mathrm{CDCl}_{3}\right): \delta=6.76$ (non-deuterated residual signal) ppm (Fig. S6). MS (ESI $): m / z$ (\%) $=308.3$ $\left(100,[\mathrm{M}+\mathrm{H}]^{+}\right)$(Fig. S7 - S8). 


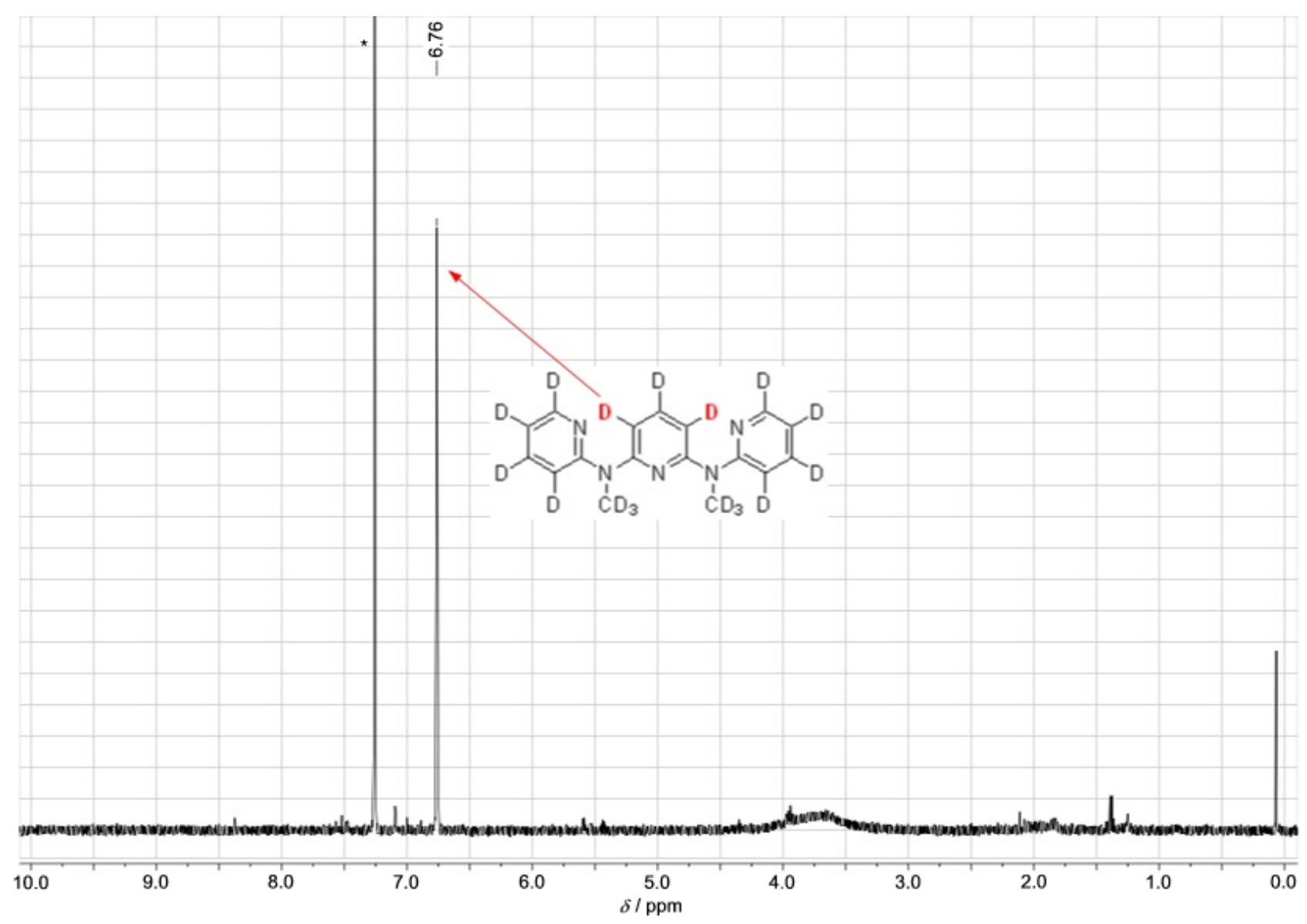

Figure S6. ${ }^{1} \mathrm{H}$ NMR spectrum ( $400 \mathrm{MHz}$ ) of $\left[\mathrm{D}_{17}\right]$-ddpd in $\mathrm{CDCl}_{3}$ showing the only major residual proton resonance due to incomplete deuteration at the position indicated in bold red. The asterisk marks residual solvent resonance.

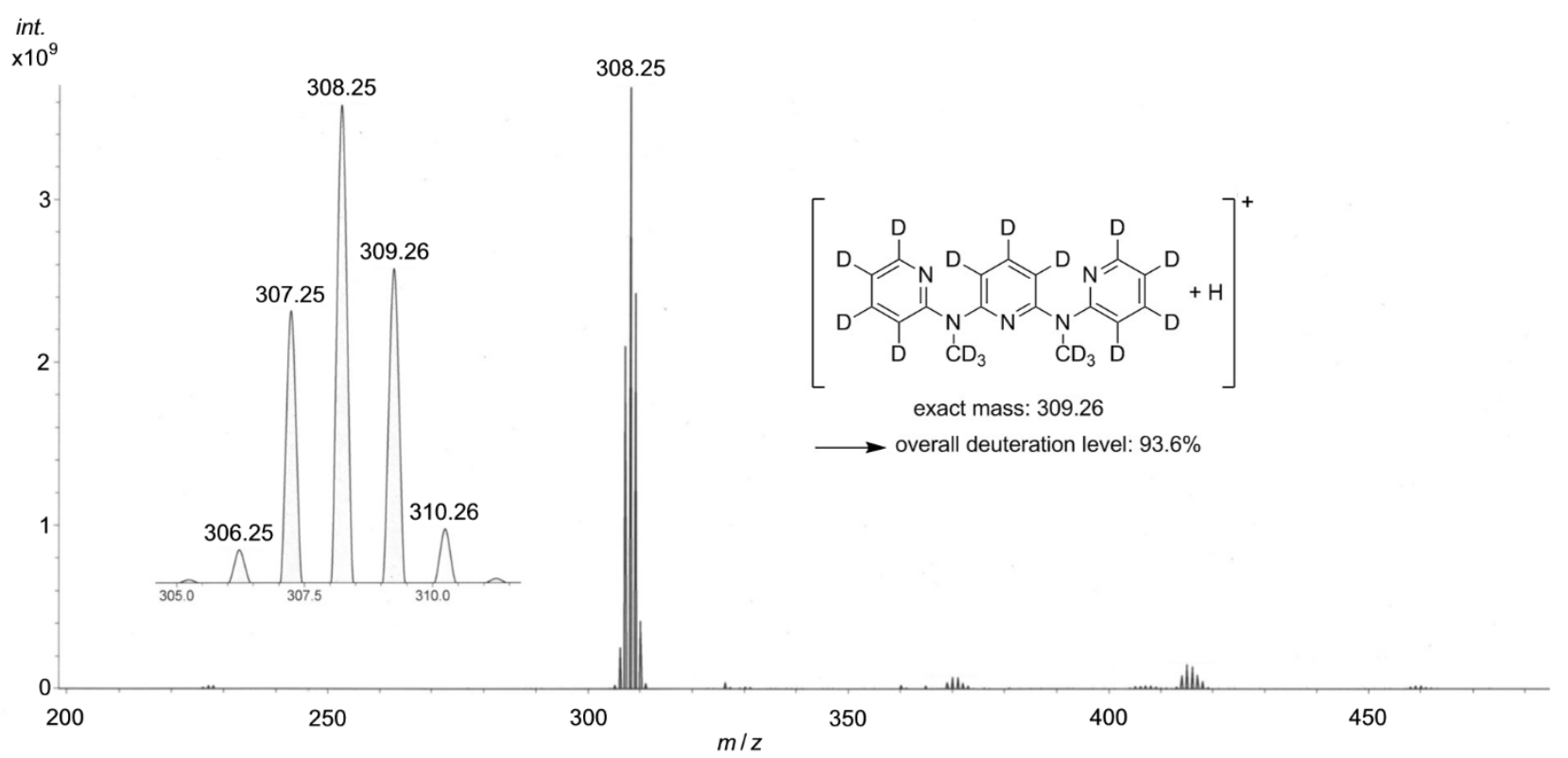

Figure S7. $\mathrm{ESI}^{+}$mass spectrum of $\left[\mathrm{D}_{17}\right]$-ddpd. 


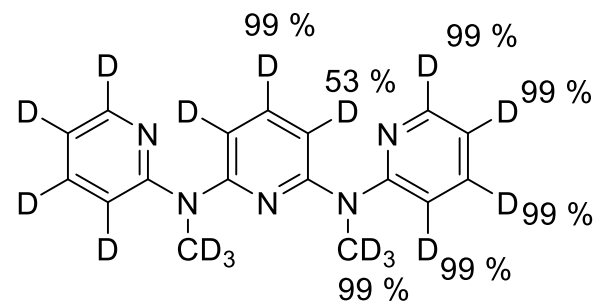

Figure S8. Detailed deuteration pattern of $\left[\mathrm{D}_{17}\right]$-ddpd from combined ${ }^{1} \mathrm{H}$ NMR and mass spectrometry data.

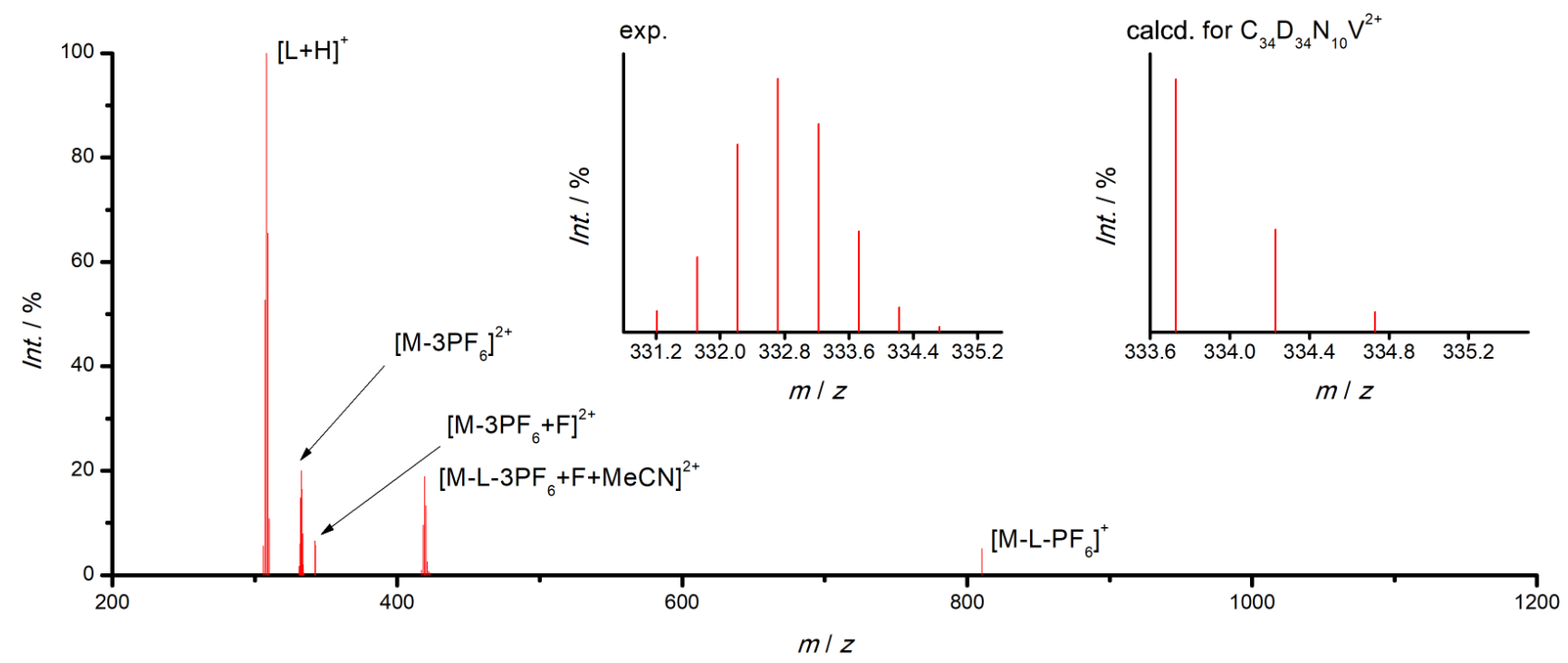

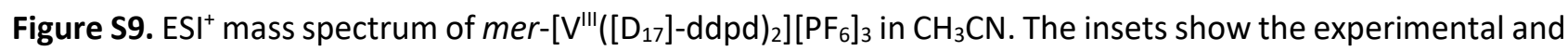
calculated isotopic pattern of the $\left[\mathrm{M}-3 \mathrm{PF}_{6}\right]^{2+}$ peak. 
Synthesis of $\left[\mathbf{V}(\mathbf{d d p d})_{2}\right]\left[\mathrm{PF}_{6}\right]_{3}:\left[\mathrm{V}(\mathrm{NCMe})_{6}\right]\left[\mathrm{BPh}_{4}\right]_{2}(42.0 \mathrm{mg}, 0.05 \mathrm{mmol}, 1.0 \mathrm{eq})$ was dissolved in acetonitrile $(4 \mathrm{ml})$ and $\left[{ }^{n} \mathrm{Bu}_{4} \mathrm{~N}\right]\left[\mathrm{PF}_{6}\right](52.2 \mathrm{mg}, 0.14 \mathrm{mmol}, 2.8$ eq.), dissolved in acetonitrile $(2 \mathrm{ml})$, was added under stirring. The ligand ddpd ( $27.7 \mathrm{mg}, 0.09 \mathrm{mmol}, 1.8 \mathrm{eq}$.$) , dissolved in acetonitrile (2 \mathrm{ml})$, was added. The turquoise solution was stirred for $18 \mathrm{~h}$ and turned dark red within the first several hours. Upon addition of ferrocenium hexafluorophosphate $(15.0 \mathrm{mg}, 0.05 \mathrm{mmol}, 1.0$ eq.), dissolved in acetonitrile $(4 \mathrm{ml})$, the red solution turned green. The solvent was removed under reduced pressure and the dark green residue was washed with $2 \mathrm{ml}$ aliquots of petroleum ether (40/60) until the washing solution remained colorless. After dissolving the green product in acetonitrile $(4 \mathrm{ml})$, black crystals were obtained from slow diffusion of diethyl ether into the solution. Yield: $18.2 \mathrm{mg}(0.02 \mathrm{mmol}, 44 \%) . \mathrm{C}_{34} \mathrm{H}_{34} \mathrm{~F}_{18} \mathrm{~N}_{10} \mathrm{P} \mathrm{P}_{3} \mathrm{~V}$ : calcd. C $38.22 ; \mathrm{H} 3.21 ; \mathrm{N} 13.11 \%$; found: C 38.22; H 3.43; N $13.56 \%$. IR (ATR): $\tilde{v}=1607$ (m), 1581 (w), 1568 (w), $1494(\mathrm{~m}), 1449$ (w), 1432 (m), $1364(w), 1341(w), 1319(w), 1240(w), 1201(w), 1175(w), 1138$ (w), $1093(w), 1065$ (w), 1052 (w, sh), 1020 (w), $1011(w), 949(w), 876(w), 832(s), 807(s), 775(s), 750(m), 716(w), 656(w), 633(w), 582(w), 556(s)$ $523(w), 507(w), 480(w), 467(w), 449(w), 436(w) \mathrm{cm}^{-1} . \mathrm{MS}\left(\mathrm{ESI}^{+}, \mathrm{CH}_{3} \mathrm{CN}\right): \mathrm{m} / z(\%)=316.6(35)\left[\mathrm{M}-3 \mathrm{PF}_{6}\right]^{3+}$, $326.1(5)\left[\mathrm{M}-3 \mathrm{PF}_{6}+\mathrm{F}\right]^{+}, 778.2(100)\left[\mathrm{M}-2 \mathrm{PF}_{6}\right]^{+} . \mathrm{UV} / \mathrm{VIS} / \mathrm{NIR}\left(\mathrm{CH}_{3} \mathrm{CN}\right): \lambda\left(\varepsilon / \mathrm{M}^{-1} \mathrm{~cm}^{-1}\right)=207$ (25015), 241

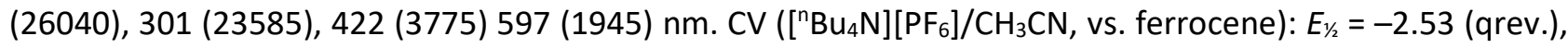
-2.31 (qrev.), -0.275 (rev.) V; $E_{\mathrm{p}}=1.74$ (irrev.), 1.86 (irrev.) V.

Synthesis of $\left[\mathbf{V}\left(\left[\mathbf{D}_{17}\right]-\mathbf{d d p d}\right)_{2}\right]\left[\mathbf{P F}_{6}\right]_{3}$ : The deuterated complex was prepared analogously, starting from $\left[\mathbf{D}_{17}\right]-$ ddpd (27.7 mg, $0.09 \mathrm{mmol}, 2.0$ eq.). Yield: $8.6 \mathrm{mg}(0.008 \mathrm{mmol}, 13 \%)$. The degree of deuteration was fully retained according to $\mathrm{ESI}^{+}$mass spectrometry. 
Table S1. Bond distances / $\AA$ and angles / ${ }^{\circ}$ of mer-[V'II (ddpd) $\left.)_{2}\right]\left[\mathrm{PF}_{6}\right]_{3} \times 3 \mathrm{CH}_{3} \mathrm{CN}(\mathrm{XRD})$ and $m e r-\left[\mathrm{V}^{\prime \prime \prime}(\mathrm{ddpd})_{2}\right]^{3+}$ (DFT-UKS).

\begin{tabular}{|l|l|l|l|l|l|}
\hline & \multicolumn{2}{|c|}{ ddpd(A) } & \multicolumn{2}{c|}{ ddpd(B) } & \\
\hline \multirow{5}{*}{} & V-N1 / & N1-V-N2 / & V-N4 / & $\begin{array}{l}\text { N4-V-N5 / } \\
\text { N4-V-N6 / }\end{array}$ & shape \\
& V-N2 / & N1-V-N3 / & V-N5 / & Nameter \\
& V-N3 & N2-V-N3 & V-N6 & N5-V-N6 & S(OC-6) \\
\hline XRD & $2.079(2) /$ & $84.14(6) /$ & $2.074(2) /$ & $85.12(6) /$ & 0.63 \\
& $2.062(1) /$ & $168.23(6) /$ & $2.058(1) /$ & $169.73(6) /$ & \\
& $2.068(2)$ & $84.13(6)$ & $2.081(2)$ & $84.69(6)$ & \\
\hline DFT-UKS & 2.095 & 84.24 & 2.092 & 84.16 & 0.68 \\
& 2.078 & 168.56 & 2.077 & 168.40 & \\
& 2.097 & 84.32 & 2.096 & 84.25 & \\
\hline
\end{tabular}

Table S2. Cartesian Coordinates of the DFT-UKS calculated ground state geometry of $m e r$ - $\left[\mathrm{V}^{\mathrm{III}}(\mathrm{ddpd})_{2}\right]^{3+}$.

\begin{tabular}{|c|c|}
\hline 23 & -0.436272000 \\
\hline 7 & 0.057762000 \\
\hline 7 & 0.566002000 \\
\hline 7 & 1.171885000 \\
\hline 7 & 1.735378000 \\
\hline 7 & -0.608309000 \\
\hline 7 & -1.881315000 \\
\hline 7 & -2.967475000 \\
\hline 7 & -2.043433000 \\
\hline 7 & -1.078209000 \\
\hline 7 & 0.685217000 \\
\hline 6 & -0.136059000 \\
\hline 1 & -0.259546000 \\
\hline 6 & -0.211793000 \\
\hline 1 & -0.382655000 \\
\hline 6 & -0.081057000 \\
\hline 1 & -0.146249000 \\
\hline 6 & 0.163009000 \\
\hline 1 & 0.313028000 \\
\hline 6 & 0.261183000 \\
\hline 6 & 0.413147000 \\
\hline 1 & -0.540605000 \\
\hline 1 & 1.213525000 \\
\hline 1 & 0.400412000 \\
\hline 6 & 1.420814000 \\
\hline 6 & 2.497005000 \\
\hline 1 & 2.690014000 \\
\hline 6 & 3.346353000 \\
\hline 1 & 4.198093000 \\
\hline 6 & 3.106104000 \\
\hline 1 & 3.743171000 \\
\hline 6 & 2.000162000 \\
\hline 6 & 2.891430000 \\
\hline 1 & 2.632379000 \\
\hline 1 & 3.191768000 \\
\hline 1 & 3.718554000 \\
\hline 6 & 0.483182000 \\
\hline 6 & 0.357628000 \\
\hline 1 & 1.231585000 \\
\hline 6 & -0.902124000 \\
\hline 1 & -1.006918000 \\
\hline 6 & -2.027893000 \\
\hline 1 & -3.027361000 \\
\hline 6 & -1.836368000 \\
\hline 1 & -2.670761000 \\
\hline 6 & -1.729583000 \\
\hline 1 & -1.035394000 \\
\hline 6 & -2.384217000 \\
\hline 1 & -2.220705000 \\
\hline 6 & -3.239090000 \\
\hline 1 & -3.767402000 \\
\hline 6 & -3.435458000 \\
\hline 1 & -4.127804000 \\
\hline 6 & -2.758753000 \\
\hline 6 & -3.604148000 \\
\hline 1 & -3.131234000 \\
\hline 1 & -4.676344000 \\
\hline 1 & -3.427930000 \\
\hline 6 & -3.045409000 \\
\hline 6 & -4.137792000 \\
\hline 1 & -4.931914000 \\
\hline 6 & -4.214122000 \\
\hline 1 & -5.0639 \\
\hline
\end{tabular}

\begin{tabular}{|c|c|}
\hline 4. 887622000 & 1.4985860 \\
\hline 4.359394000 & 3.4653440 \\
\hline 2.182490000 & 2.743718 \\
\hline 3.674479000 & 0.990576 \\
\hline 5.193304000 & -0.753156 \\
\hline 5.160298000 & -0.568481 \\
\hline 3.370031000 & 1.418202 \\
\hline .322157000 & 3.270756 \\
\hline 6.107196000 & 1.995698 \\
\hline 7.861890000 & 0.71 \\
\hline 6.647703000 & \\
\hline 5.257662000 & 4.457962 \\
\hline 6.284304000 & 4.150607 \\
\hline 4.895243000 & 5.778999 \\
\hline 5.644256000 & 6.5364280 \\
\hline 3.543448000 & 6.0970410 \\
\hline 3.212495000 & 7.123799 \\
\hline 2.623064000 & 5.098671 \\
\hline 1.585384000 & 5.342659 \\
\hline .065063000 & \\
\hline 0.745847000 & 3.0142000 \\
\hline 0.588956000 & 3.509025 \\
\hline 0.349688000 & 3.6390440 \\
\hline 0.219756000 & 2.0658430 \\
\hline 2.536984000 & 1.6907070 \\
\hline 1.718823000 & 1.3814360 \\
\hline 0.831463000 & 1.9598050 \\
\hline 2.091812000 & 0.355262 \\
\hline 1.474345000 & 0.1084270 \\
\hline 3.253067000 & -0.3 \\
\hline 3.535313000 & -1.1770840 \\
\hline 4.024811000 & -0.0282030 \\
\hline 5.883199000 & -1.3441840 \\
\hline 6.928522000 & -1.4834880 \\
\hline 5.454711000 & -2.3003060 \\
\hline 5.823470000 & -0.6451150 \\
\hline 5.466337000 & -1.2939280 \\
\hline 6.053399000 & -2.558570 \\
\hline 6.307009000 & -3.13 \\
\hline 6.260699000 & -3.0812590 \\
\hline 6.701378000 & -4.0624680 \\
\hline 5.874740000 & -2.3535870 \\
\hline 6.005690000 & -2.7380810 \\
\hline 5.328887000 & -1.1098260 \\
\hline 5.035170000 & -0.4925010 \\
\hline 2.362329000 & 0.5289060 \\
\hline 2.541877000 & -0.277137 \\
\hline 1.162843000 & 0.65140 \\
\hline 0.380897000 & -0.073714 \\
\hline 0.990094000 & 1.7397950 \\
\hline 0.057720000 & 1.8796550 \\
\hline 2.025466000 & 2.6303700 \\
\hline 1.914655000 & 3.4472430 \\
\hline 3.234073000 & 2.4297240 \\
\hline 4.064075000 & 4.5704700 \\
\hline 3.195145000 & 5.0183790 \\
\hline 3.887573000 & 4.4859730 \\
\hline 4.921238000 & 5.211336 \\
\hline 5.633621000 & 2.7816360 \\
\hline 6.421152000 & 3.112467 \\
\hline 6.021879000 & 3.7201810 \\
\hline 7.705453000 & 2.603668 \\
\hline 329648000 & \\
\hline
\end{tabular}




$\begin{array}{rrr}-3.204065000 & 8.191998000 & 1.793981000 \\ -3.239019000 & 9.200534000 & 1.418905000 \\ -2.117001000 & 7.375772000 & 1.514158000 \\ -1.433285000 & 8.883518000 & -0.284577000 \\ -0.691772000 & 8.861308000 & -1.077409000 \\ -1.478835000 & 9.886173000 & 0.140329000 \\ -2.398162000 & 8.626338000 & -0.708437000 \\ 0.257173000 & 7.779071000 & 1.094684000 \\ 1.137328000 & 8.843198000 & 0.867841000 \\ 0.793923000 & 9.744769000 & 0.390211000 \\ 1.954271000 & 6.577639000 & 2.147616000 \\ 2.440965000 & 8.742728000 & 1.308045000 \\ 2.860348000 & 7.594130000 & 1.978984000 \\ 2.229881000 & 5.657694000 & 2.639060000 \\ 3.867457000 & 7.488226000 & 2.351171000 \\ 3.123251000 & 9.565649000 & 1.149268000\end{array}$

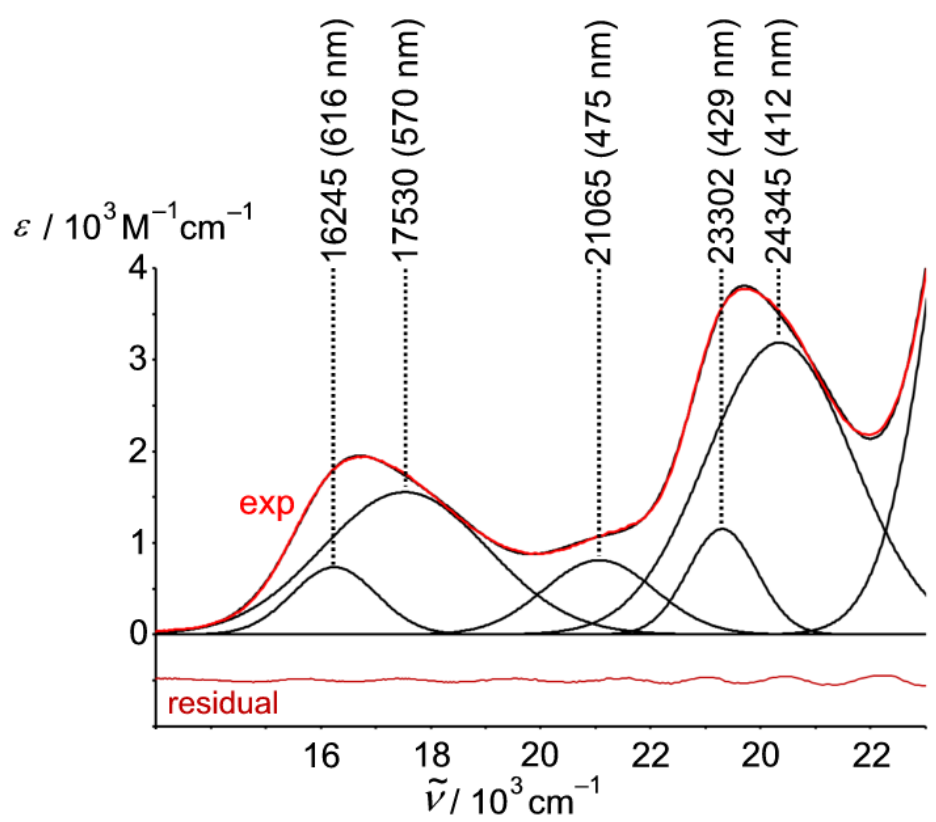

Figure S10. UV/Vis spectrum of mer- $\left[\mathrm{V}^{\prime \prime \prime}(\mathrm{ddpd})_{2}\right]\left[\mathrm{PF}_{6}\right]_{3}$ in $\mathrm{CH}_{3} \mathrm{CN}$ (red) including Gaussian deconvolution (black). 
Table S3. TD-DFT calculated spin-allowed transitions (UKS) and triplet states (RKS). The assignment of the UKS states was accomplished by visual inspection of difference electron densities (Table S4). The assignment of the RKS states corresponds to the largest contribution to the charge transfer number (Fig. S21).

\begin{tabular}{|c|c|c|c|c|c|c|c|c|c|}
\hline \multicolumn{6}{|c|}{ TD-DFT-UKS } & \multicolumn{4}{|c|}{ TD-DFT-RKS } \\
\hline state & $\tilde{v} / \mathrm{cm}^{-1}$ & $\lambda / \mathrm{nm}$ & $E / \mathrm{eV}$ & $f_{\text {osc }}$ & assignment & $\tilde{v} / \mathrm{cm}^{-1}$ & $\lambda / \mathrm{nm}$ & $E / \mathrm{eV}$ & assignment \\
\hline 1 & 3589 & 2786 & 0.445 & $7.71 \mathrm{E}-04$ & ${ }^{3} \mathrm{MC}$ & 2084 & 4800 & 0.258 & ${ }^{3} \mathrm{MC}$ \\
\hline 2 & 5077 & 1970 & 0.629 & $7.41 \mathrm{E}-06$ & ${ }^{3} \mathrm{MC}$ & & & & \\
\hline 3 & 15858 & 631 & 1.966 & $1.31 \mathrm{E}-02$ & ${ }^{3} \mathrm{LMCT}(\mathrm{NMe} \rightarrow \mathrm{V})$ & & & & \\
\hline 4 & 16237 & 616 & 2.013 & $9.71 \mathrm{E}-04$ & ${ }^{3} \mathrm{LMCT}(\mathrm{NMe} \rightarrow \mathrm{V})$ & & & & \\
\hline 5 & 19875 & 503 & 2.464 & $3.64 \mathrm{E}-03$ & ${ }^{3} \mathrm{LMCT}(\mathrm{NMe} \rightarrow \mathrm{V})$ & & & & \\
\hline 6 & 20343 & 492 & 2.522 & $3.32 \mathrm{E}-06$ & ${ }^{3} \mathrm{LMCT}(\mathrm{NMe} \rightarrow \mathrm{V})$ & & & & \\
\hline 7 & 22097 & 453 & 2.740 & $2.93 \mathrm{E}-02$ & ${ }^{3} \mathrm{LMCT}(\mathrm{NMe} \rightarrow \mathrm{V})$ & & & & \\
\hline 8 & 22543 & 444 & 2.795 & $1.20 \mathrm{E}-03$ & ${ }^{3} \mathrm{LMCT}(\mathrm{NMe} \rightarrow \mathrm{V})$ & & & & \\
\hline 9 & 22675 & 441 & 2.811 & $2.66 \mathrm{E}-04$ & ${ }^{3} \mathrm{MC}$ & 22529 & 444 & 2.793 & ${ }^{3} \mathrm{MC}$ \\
\hline 10 & 24203 & 413 & 3.001 & $2.42 \mathrm{E}-06$ & ${ }^{3} \mathrm{MC}$ & 24786 & 404 & 3.073 & ${ }^{3} \mathrm{MC}$ \\
\hline 11 & 24582 & 407 & 3.048 & $1.62 \mathrm{E}-04$ & ${ }^{3} \mathrm{LMCT}(\mathrm{NMe} \rightarrow \mathrm{V})$ & 26901 & 372 & 3.335 & ${ }^{3} \mathrm{LMCT}$ \\
\hline 12 & 24875 & 402 & 3.084 & $1.12 \mathrm{E}-02$ & ${ }^{3} \mathrm{LMCT}(\mathrm{NMe} \rightarrow \mathrm{V})$ & 27316 & 366 & 3.387 & ${ }^{3} \mathrm{LMCT}$ \\
\hline 13 & 26190 & 382 & 3.247 & 7.63E-04 & ${ }^{3} \mathrm{LMCT}(\mathrm{NMe} \rightarrow \mathrm{V}) /{ }^{3} \mathrm{MC}$ & 27616 & 362 & 3.424 & ${ }^{3} \mathrm{LMCT}$ \\
\hline 14 & 26214 & 382 & 3.250 & $3.31 \mathrm{E}-04$ & ${ }^{3} \mathrm{MC} / \mathrm{LMCT}(\mathrm{NMe} \rightarrow \mathrm{V})$ & & & & \\
\hline 15 & 26329 & 380 & 3.264 & $1.01 \mathrm{E}-04$ & ${ }^{3} \mathrm{LMCT}(\mathrm{NMe} \rightarrow \mathrm{V})$ & & & & \\
\hline 16 & 26661 & 375 & 3.306 & $4.13 \mathrm{E}-04$ & ${ }^{3} \mathrm{MC} /{ }^{3} \mathrm{LMCT}(\mathrm{NMe} \rightarrow \mathrm{V})$ & 28058 & 356 & 3.479 & ${ }^{3} \mathrm{LMCT}$ \\
\hline 17 & 26932 & 371 & 3.339 & $3.68 \mathrm{E}-04$ & ${ }^{3} \mathrm{MC} /{ }^{3} \mathrm{LMCT}(\mathrm{NMe} \rightarrow \mathrm{V})$ & 30461 & 328 & 3.777 & ${ }^{3} \mathrm{LMCT}$ \\
\hline 18 & 27056 & 370 & 3.355 & $8.04 \mathrm{E}-07$ & ${ }^{3} \mathrm{MC} /{ }^{3} \mathrm{LMCT}(\mathrm{NMe} \rightarrow \mathrm{V})$ & 30586 & 327 & 3.792 & ${ }^{3} \mathrm{LMCT}$ \\
\hline 19 & 27374 & 365 & 3.394 & $2.14 \mathrm{E}-03$ & ${ }^{3} \mathrm{MC}$ & 31428 & 318 & 3.897 & ${ }^{3} \mathrm{LMCT}$ \\
\hline 20 & 27914 & 358 & 3.461 & $2.08 \mathrm{E}-04$ & ${ }^{3} \mathrm{LMCT}(\mathrm{NMe} \rightarrow \mathrm{V})$ & 31650 & 316 & 3.924 & ${ }^{3} \mathrm{LMCT}$ \\
\hline 21 & 28340 & 353 & 3.514 & $3.22 \mathrm{E}-04$ & ${ }^{3} \mathrm{LMCT}(\mathrm{NMe} \rightarrow \mathrm{V})$ & & & & \\
\hline 22 & 28285 & 354 & 3.507 & $6.86 \mathrm{E}-03$ & ${ }^{3} \mathrm{LMCT}(\mathrm{NMe} \rightarrow \mathrm{V}) /{ }^{3} \mid \mathrm{LCT}$ & & & & \\
\hline 23 & 28368 & 353 & 3.517 & $2.12 \mathrm{E}-03$ & ${ }^{3} \mathrm{LMCT}(\mathrm{NMe} \rightarrow \mathrm{V}) /{ }^{3} \mid \mathrm{LCT}$ & & & & \\
\hline 24 & 29010 & 345 & 3.597 & $1.71 \mathrm{E}-02$ & ${ }^{3} \mathrm{LMCT}(\mathrm{NMe} \rightarrow \mathrm{V}) /{ }^{3} \mid \mathrm{LCT}$ & & & & \\
\hline 25 & 29431 & 340 & 3.649 & $2.62 \mathrm{E}-02$ & ${ }^{3} \mathrm{LMCT}(\mathrm{NMe} \rightarrow \mathrm{V}) /^{3} \mathrm{ILCT}$ & & & & \\
\hline 26 & 30068 & 333 & 3.728 & 4.04E-03 & ${ }^{3} \mathrm{MC}$ & & & & \\
\hline 27 & 30568 & 327 & 3.790 & $1.51 \mathrm{E}-04$ & ${ }^{3} \mathrm{LMCT}(\mathrm{NMe} \rightarrow \mathrm{V})$ & & & & \\
\hline 28 & 30709 & 326 & 3.807 & $9.10 \mathrm{E}-05$ & ${ }^{3} \mathrm{LMCT}(\mathrm{NMe} \rightarrow \mathrm{V}) /{ }^{3} \mathrm{ILCT}$ & & & & \\
\hline 29 & 30659 & 326 & 3.801 & $3.28 \mathrm{E}-04$ & ${ }^{3} \mathrm{LMCT}(\mathrm{NMe} \rightarrow \mathrm{V}) /^{3} \mathrm{ILCT}$ & & & & \\
\hline 30 & 30835 & 324 & 3.823 & $1.10 \mathrm{E}-02$ & ${ }^{3} \mathrm{LMCT}(\mathrm{NMe} \rightarrow \mathrm{V}) /{ }^{3} \mathrm{ILCT}$ & & & & \\
\hline 31 & 30814 & 325 & 3.820 & $2.33 \mathrm{E}-04$ & ${ }^{3} \mathrm{LMCT}(\mathrm{NMe} \rightarrow \mathrm{V}) /^{3} \mathrm{ILCT}$ & & & & \\
\hline 32 & 31164 & 321 & 3.864 & 3.39E-02 & ${ }^{3} \mathrm{LMCT}(\mathrm{NMe} \rightarrow \mathrm{V}) /^{3} \mathrm{ILCT}$ & & & & \\
\hline 33 & 31585 & 317 & 3.916 & $1.27 \mathrm{E}-04$ & ${ }^{3} \mathrm{LMCT}(\mathrm{NMe} \rightarrow \mathrm{V})$ & & & & \\
\hline 34 & 31725 & 315 & 3.933 & $1.19 \mathrm{E}-04$ & ${ }^{3} \mathrm{LMCT}(\mathrm{NMe} \rightarrow \mathrm{V}) /{ }^{3} \mathrm{ILCT}$ & & & & \\
\hline 35 & 31708 & 315 & 3.931 & $5.79 \mathrm{E}-03$ & ${ }^{3} \mathrm{LMCT}(\mathrm{NMe} \rightarrow \mathrm{V}) /{ }^{3} \mathrm{ILCT}$ & & & & \\
\hline 36 & 31789 & 315 & 3.941 & $4.03 E-03$ & ${ }^{3} \mathrm{LMCT}(\mathrm{NMe} \rightarrow \mathrm{V})$ & & & & \\
\hline 37 & 31794 & 315 & 3.942 & $2.68 \mathrm{E}-03$ & ${ }^{3} \mathrm{LMCT}(\mathrm{NMe} \rightarrow \mathrm{V})$ & & & & \\
\hline 38 & 31985 & 313 & 3.966 & $2.60 \mathrm{E}-02$ & ${ }^{3} \mathrm{LMCT}(\mathrm{NMe} \rightarrow \mathrm{V}) /{ }^{3} \mathrm{ILCT}$ & & & & \\
\hline 39 & 32267 & 310 & 4.001 & $7.96 \mathrm{E}-04$ & ${ }^{3} \mathrm{LMCT}(\mathrm{NMe} \rightarrow \mathrm{V})$ & & & & \\
\hline 40 & 32293 & 310 & 4.004 & $5.48 \mathrm{E}-02$ & ${ }^{3} \mathrm{LMCT}(\mathrm{NMe} \rightarrow \mathrm{V}) /^{3} \mathrm{ILCT}$ & & & & \\
\hline 41 & 32487 & 308 & 4.028 & $1.44 \mathrm{E}-02$ & ${ }^{3} \mathrm{LMCT}(\mathrm{NMe} \rightarrow \mathrm{V})$ & & & & \\
\hline 42 & 32451 & 308 & 4.023 & $1.53 \mathrm{E}-03$ & ${ }^{3} \mathrm{LMCT}(\mathrm{NMe} \rightarrow \mathrm{V})$ & & & & \\
\hline 43 & 32912 & 304 & 4.081 & $3.15 \mathrm{E}-02$ & ${ }^{3} \mathrm{LMCT}(\mathrm{NMe} \rightarrow \mathrm{V})$ & & & & \\
\hline 44 & 32947 & 304 & 4.085 & $7.00 \mathrm{E}-03$ & ${ }^{3} \mathrm{LMCT}(\mathrm{NMe} \rightarrow \mathrm{V}) /{ }^{3} \mathrm{ILCT}$ & & & & \\
\hline 45 & 32928 & 304 & 4.083 & 7.16E-02 & ${ }^{3} \mathrm{LMCT}(\mathrm{NMe} \rightarrow \mathrm{V})$ & & & & \\
\hline 46 & 33231 & 301 & 4.120 & $3.10 \mathrm{E}-04$ & ${ }^{3} \mathrm{LMCT}(\mathrm{NMe} \rightarrow \mathrm{V})$ & & & & \\
\hline 47 & 33292 & 300 & 4.128 & $1.29 \mathrm{E}-02$ & ${ }^{3} \mathrm{LMCT}(\mathrm{NMe} \rightarrow \mathrm{V}) /{ }^{3} \mathrm{ILCT}$ & & & & \\
\hline 48 & 33381 & 300 & 4.139 & $3.82 \mathrm{E}-05$ & ${ }^{3} \mathrm{LMCT}(\mathrm{NMe} \rightarrow \mathrm{V})$ & & & & \\
\hline 49 & 33701 & 297 & 4.178 & $2.04 \mathrm{E}-03$ & ${ }^{3} \mathrm{MC}$ & & & & \\
\hline 50 & 33750 & 296 & 4.185 & $1.87 \mathrm{E}-02$ & ${ }^{3} \mathrm{LMCT}(\mathrm{NMe} \rightarrow \mathrm{V}) /{ }^{3} \mathrm{ILCT}$ & & & & \\
\hline
\end{tabular}


Table S4. Difference electron densities of the 50 lowest-lying TD-DFT-UKS calculated states. (isosurface value at 0.007 a.u.; purple = electron depletion; orange = electron gain; hydrogen atoms omitted).

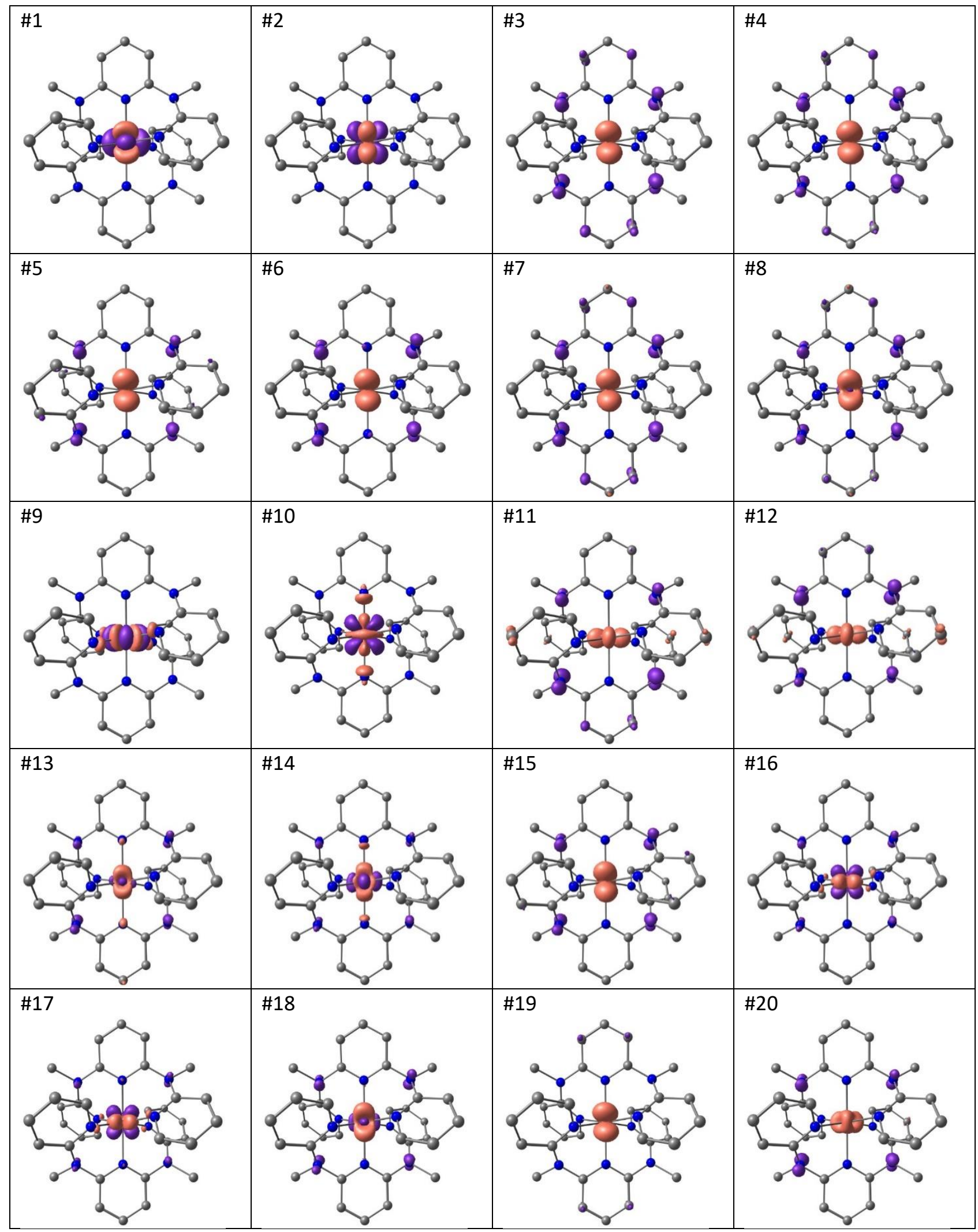




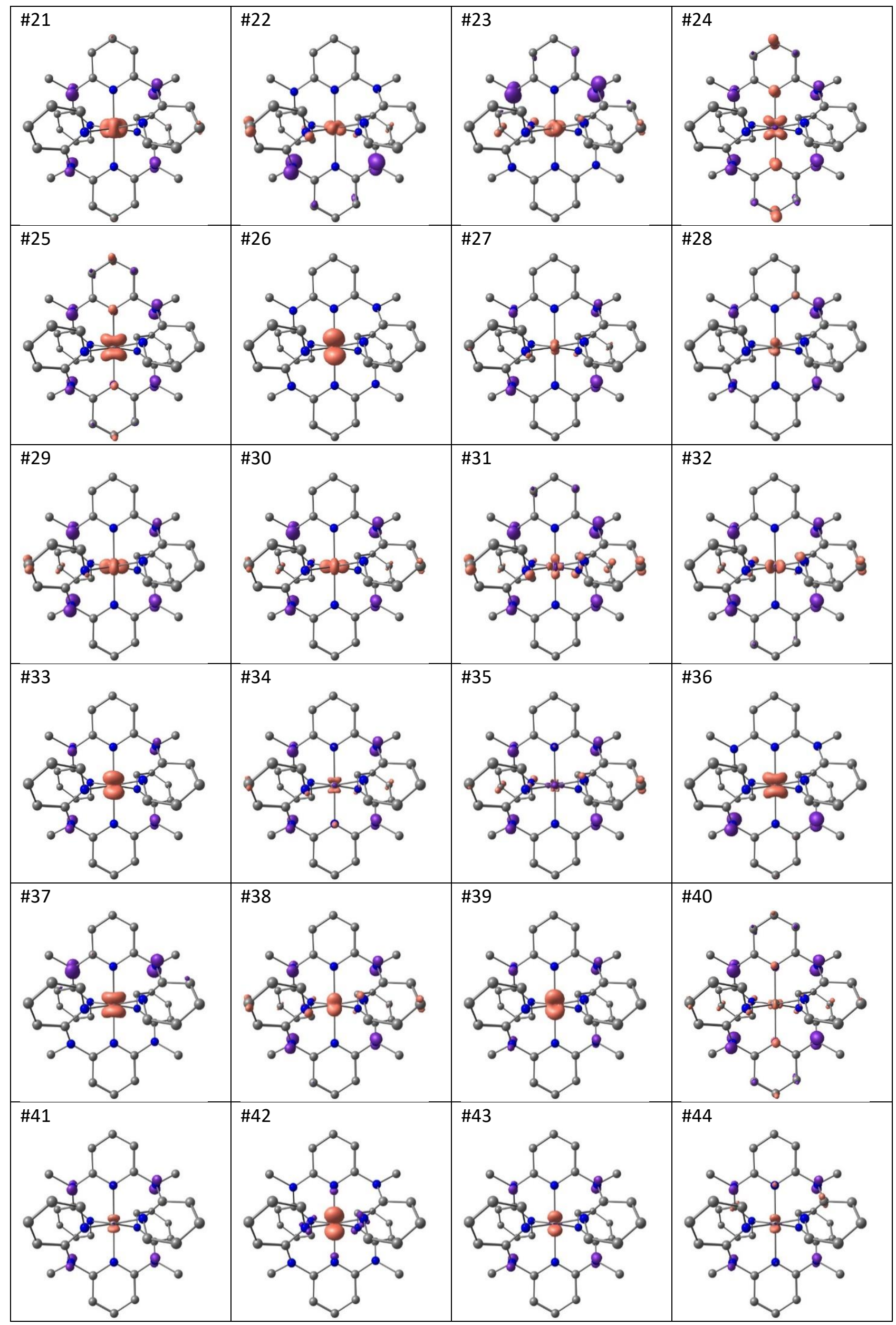




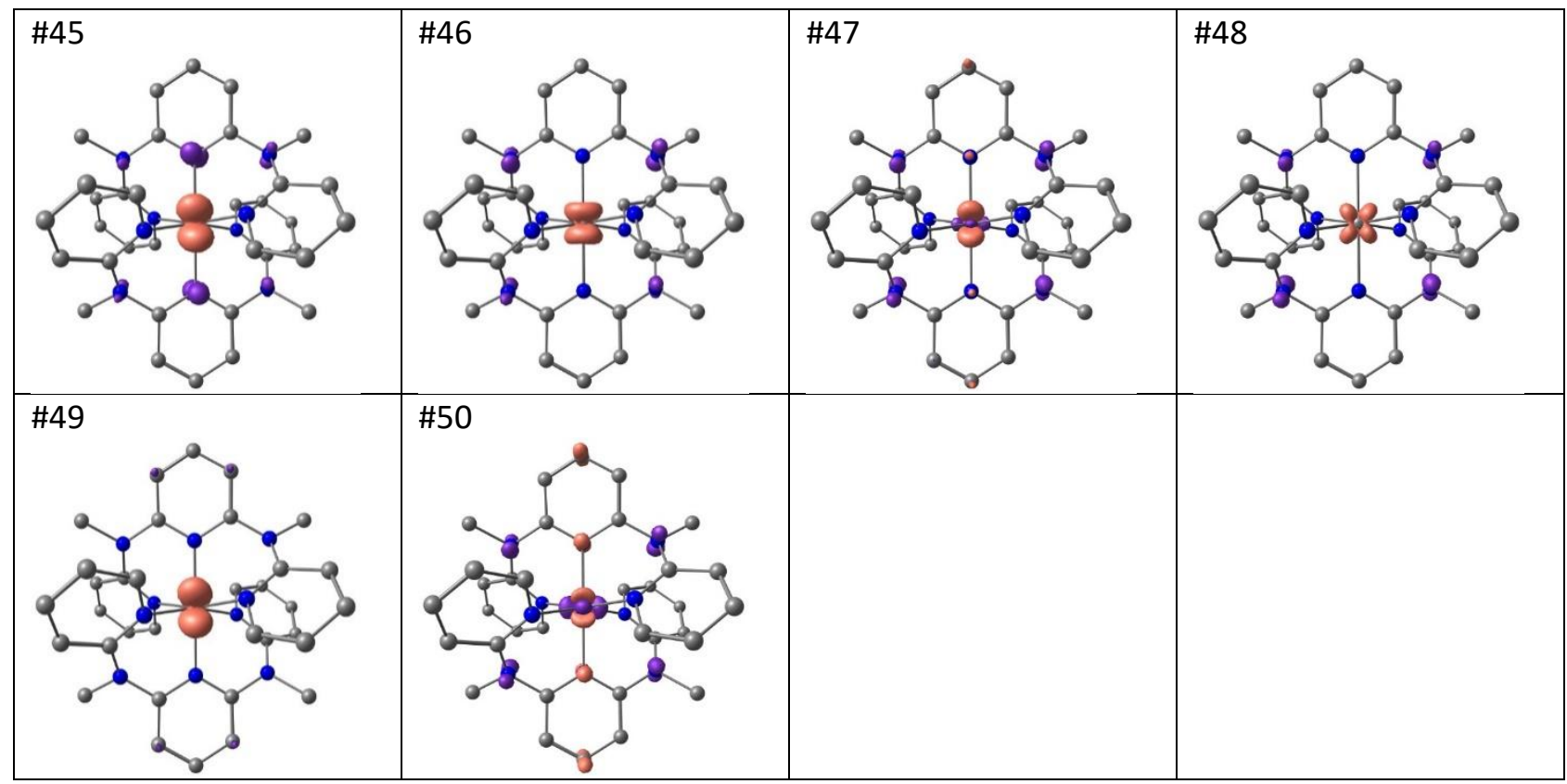


Table S5. Orbital energies/Hartree of the canonical orbitals used in the active space of the CASSCF(6,12)-FICNEVPT2 calculation, depicted at a contour value of 0.05 a.u. (hydrogen atoms omitted for clarity).

\begin{tabular}{|c|c|c|c|c|c|}
\hline \# & $E / H$ & orbital & $\#$ & $E / H$ & orbital \\
\hline 161 & -0.59153 & & 167 & +0.12076 & \\
\hline 162 & -0.59466 & & 168 & +0.8329 & \\
\hline 163 & -0.00153 & & 169 & +0.85194 & \\
\hline 164 & +0.00038 & & 170 & +0.89659 & \\
\hline 165 & +0.00753 & & 171 & +1.31242 & \\
\hline 166 & +0.11194 & & 172 & +1.49528 & \\
\hline
\end{tabular}



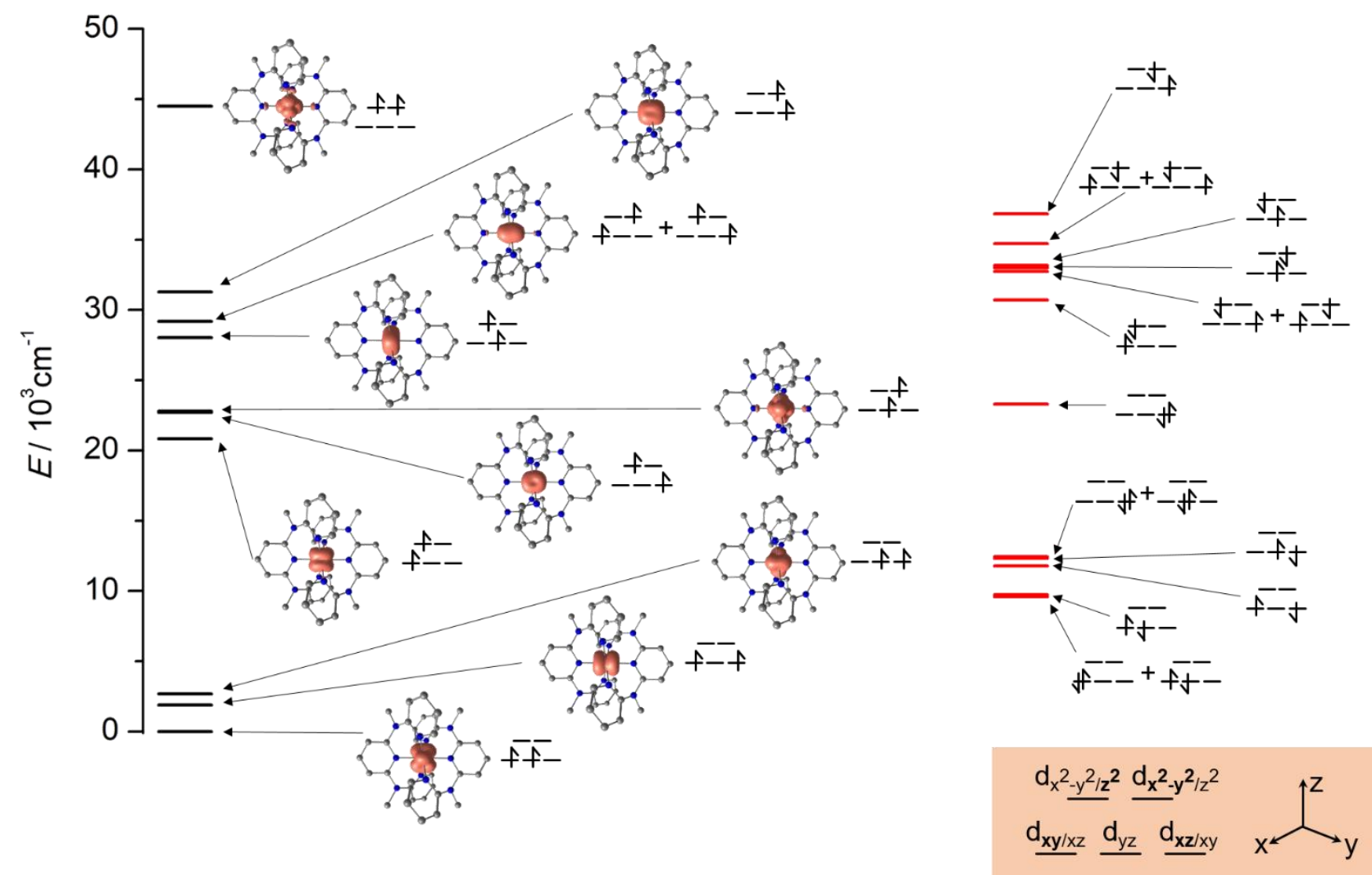

Figure S11. Energy diagram of the electronic states of $m e r-\left[V^{\prime \prime \prime}(d d p d)_{2}\right]^{3+}$ constructed from CASSCF $(6,12)$-FICNEVPT2 energies with spin densities in orange ( 0.05 a.u. isosurface value, hydrogen atoms omitted for clarity, triplet states in black, singlet states in red; a coordinate system referring to the displayed structures and $d$ orbital labels is shown in the orange box). 
Table S6. CASSCF(6,12)-FIC-NEVPT2 state compositions (the configurations are the occupation numbers for the individual active orbitals in rising energy (in $\mathrm{eV}$ and $\mathrm{cm}^{-1}$ ). The first two numbers of the configuration represent the ligand-metal $\sigma$-bonding orbitals, the next five the metal $3 d$ orbitals and the last five the $4 d$ orbitals (Table S6). Singlet states shown in red, triplet states shown in black.

\begin{tabular}{|c|c|c|c|c|c|r|r|}
\hline State & root & mult & configuration & weight & $\Delta E / \mathbf{e V}$ & $\widetilde{\boldsymbol{v}} / \mathbf{c m}^{\mathbf{- 1}}$ & $\lambda / \mathbf{n m}$ \\
\hline 0 & 0 & 3 & 221100000000 & 0.94971 & 0 & & \\
\hline 1 & 1 & 3 & 221010000000 & 0.94790 & 0.232 & 1873 & 5344 \\
\hline 2 & 2 & 3 & 220110000000 & 0.95301 & 0.333 & 2687 & 3723 \\
\hline 3 & 0 & 1 & 222000000000 & 0.65408 & 1.192 & 9612 & 1040 \\
\hline 4 & 1 & 1 & 221100000000 & 0.96124 & 1.207 & 9736 & 1027 \\
\hline 5 & 2 & 1 & 221010000000 & 0.97424 & 1.459 & 11769 & 850 \\
\hline 6 & 3 & 1 & 220110000000 & 0.95454 & 1.529 & 12333 & 811 \\
\hline 7 & 4 & 1 & 220020000000 & 0.48695 & 1.541 & 12431 & 805 \\
\hline 8 & 3 & 3 & 221001000000 & 0.83676 & 2.584 & 20844 & 480 \\
\hline 9 & 4 & 3 & 220011000000 & 0.70074 & 2.815 & 22706 & 440 \\
\hline 10 & 5 & 3 & 220100100000 & 0.42279 & 2.827 & 22803 & 439 \\
\hline 11 & 5 & 1 & 220020000000 & 0.98358 & 2.887 & 23283 & 429 \\
\hline 12 & 6 & 3 & 220101000000 & 0.94490 & 3.475 & 28026 & 357 \\
\hline 13 & 7 & 3 & 221000100000 & 0.67288 & 3.618 & 29183 & 343 \\
\hline 14 & 6 & 1 & 221001000000 & 0.91877 & 3.809 & 30720 & 326 \\
\hline 15 & 8 & 3 & 220010100000 & 0.81832 & 3.878 & 31278 & 320 \\
\hline 16 & 7 & 1 & 220011000000 & 0.57938 & 4.059 & 32735 & 305 \\
\hline 17 & 8 & 1 & 220100100000 & 0.96151 & 4.095 & 33028 & 303 \\
\hline 18 & 9 & 1 & 220101000000 & 0.97839 & 4.112 & 33163 & 302 \\
\hline 19 & 10 & 1 & 221000100000 & 0.58977 & 4.303 & 34708 & 288 \\
\hline 20 & 11 & 1 & 220010100000 & 0.93700 & 4.567 & 36835 & 271 \\
\hline 21 & 9 & 3 & 220001100000 & 0.99098 & 5.518 & 44503 & 225 \\
\hline
\end{tabular}

Table S7. Partial quantum yields $\Phi$ and luminescence lifetimes $\tau$ for $\left[\mathrm{V}(\mathrm{ddpd})_{2}\right]\left[\mathrm{PF}_{6}\right]_{3}$ and $\left[\mathrm{V}\left(\left[\mathrm{D}_{17}\right]-\mathrm{ddpd}\right)_{2}\right]\left[\mathrm{PF}_{6}\right]_{3}$ in deoxygenated solutions.

\begin{tabular}{|c|c|c|c|c|c|}
\hline compound & $\Phi_{\mathrm{F}} / \%^{\mathrm{a}}$ & $\tau_{\mathrm{F}} / \mathrm{ns}$ (fraction) $^{\mathrm{b}}$ & $\Phi_{\mathrm{p}} / \%^{\mathrm{c}}$ & $\begin{array}{c}\tau_{\mathrm{p}} / \mathrm{ns} \\
\text { (fraction) }^{\mathrm{d}}\end{array}$ & $\begin{array}{c}\tau_{\mathrm{F}} / \mathrm{ns} \\
\text { (fraction) }\end{array}$ \\
\hline & \multicolumn{3}{|c|}{ in $\mathrm{CD}_{3} \mathrm{CN}, 298 \mathrm{~K}$} & in ${ }^{\mathrm{n}} \mathrm{BuCN}, 77 \mathrm{~K}$ & in ${ }^{n} \operatorname{PrCN}, 77 \mathrm{~K}$ \\
\hline \multirow{2}{*}[\mathrm{V}(\mathrm{ddpd})_{2}]{$\left[\mathrm{PF}_{6}\right]_{3}$} & \multirow{2}{*}{2.1} & $3.2(56 \%)$ & \multirow{2}{*}{$1.8 \times 10^{-4}$} & 790 (93\%) & $1.7(51 \%)$ \\
\hline & & $8.2(44 \%)$ & & $8800(7 \%)$ & 6.3 (49 \%) \\
\hline \multirow{2}{*}[V([D_{17}]-ddpd)_{2}]{$\left[P F_{6}\right]_{3}$} & \multirow{2}{*}{1.2} & $3.3(88 \%)$ & \multirow{2}{*}{$1.6 \times 10^{-4}$} & 800 (91 \%) & \multirow[t]{2}{*}{-} \\
\hline & & $7.2(12 \%)$ & & $8300(9 \%)$ & \\
\hline
\end{tabular}

a $\lambda_{\mathrm{exc}}=308 \mathrm{~nm}, \lambda_{\mathrm{em}} \approx 340-580 \mathrm{~nm}$, measured relative to the quantum yield standard quinine in $0.1 \mathrm{M}$ aqueous $\mathrm{H}_{2} \mathrm{SO}_{4}$, estimated uncertainties $\pm 40 \%$; ${ }^{\mathrm{b}} \lambda_{\mathrm{exc}}=306 \mathrm{~nm}, \lambda_{\mathrm{em}} 390$, biexponential fitting, estimated uncertainties $\pm 10 \%$; ${ }^{c} \lambda_{\mathrm{exc}}=308 \mathrm{~nm}, \lambda_{\mathrm{em}} \approx 1050-1200 \mathrm{~nm}$, measured relative to the quantum yield standard $\left[\mathrm{Yb}(\mathrm{tta})_{3}\right.$ (phen) $]$ in toluene, estimated uncertainties $\pm 50 \%$; ${ }^{\mathrm{d}} \lambda_{\mathrm{exc}}=300 \mathrm{~nm}, \lambda_{\mathrm{em}} 1110 \mathrm{~nm}$, biexponential fitting, estimated uncertainties $\pm 20 \%$. 


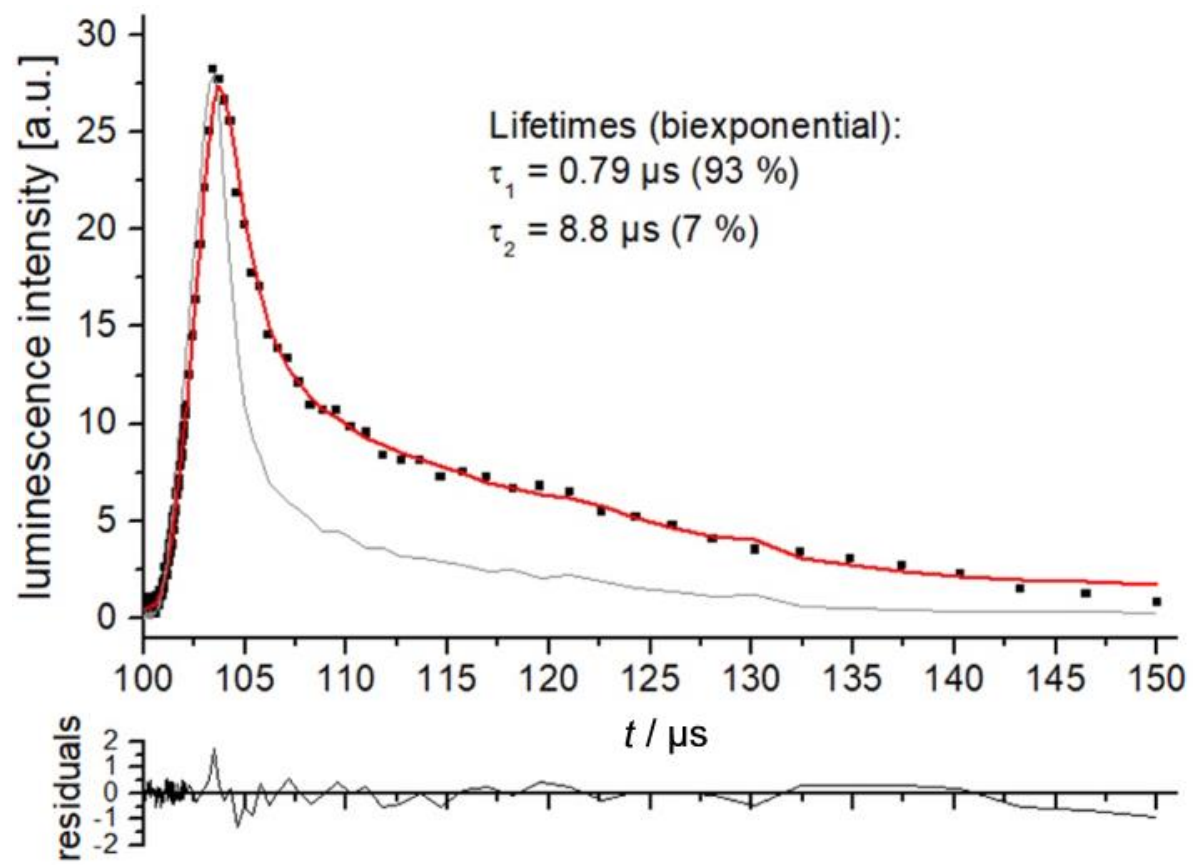

Figure S12. Luminescence decay (black scatter) for mer- $\left[\mathrm{V}(\mathrm{ddpd})_{2}\right]\left[\mathrm{PF}_{6}\right]_{3}$ in deoxygenated ${ }^{\mathrm{n}} \mathrm{BuCN}$ at $77 \mathrm{~K}$ ( $\lambda_{\mathrm{exc}}=300 \mathrm{~nm}, \lambda_{\mathrm{em}}=1110 \mathrm{~nm}$, emission path: long pass filter RG850) with biexponential fit function (red) and instrument response function (gray). 
a)
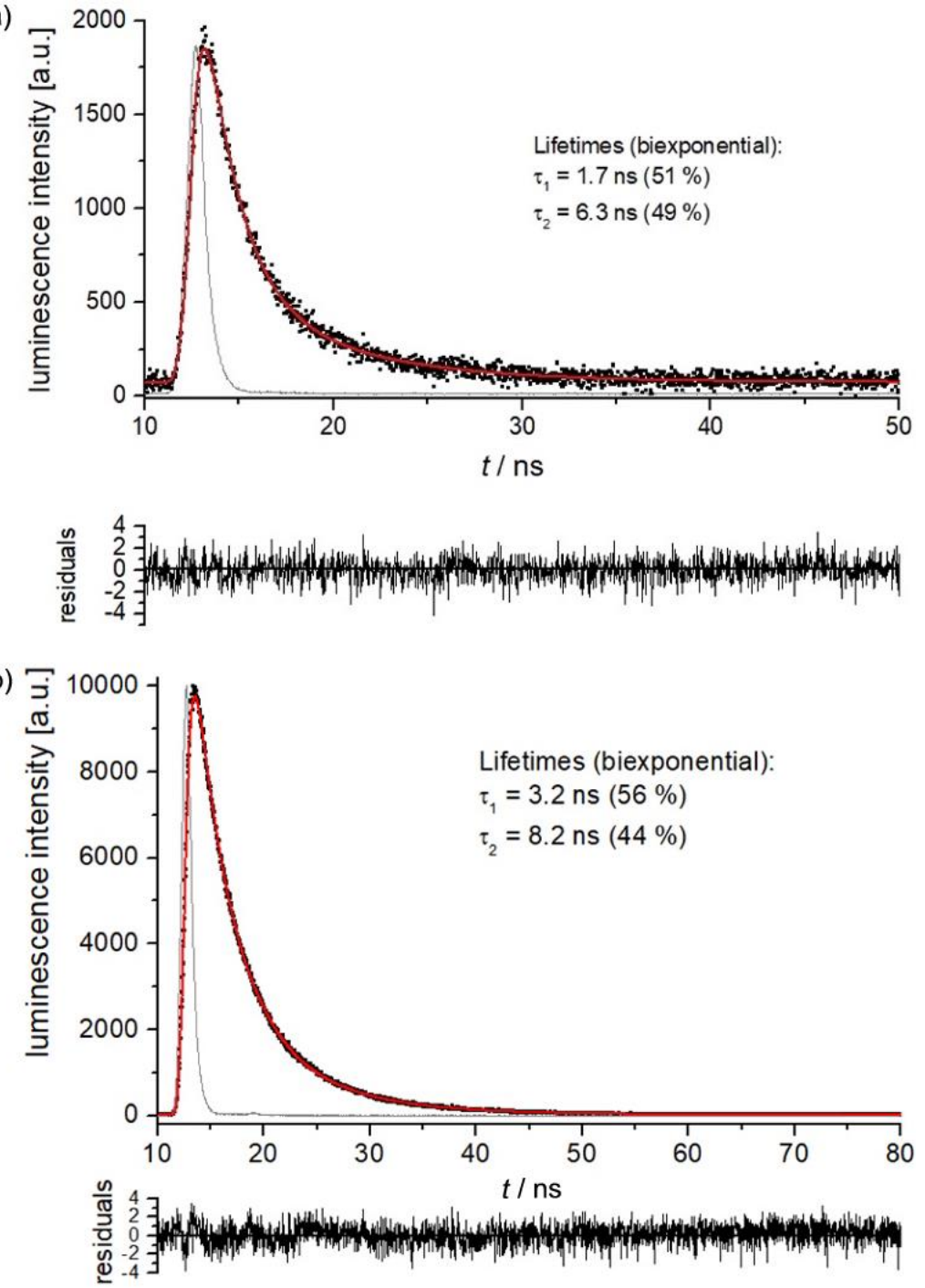

Figure S13. a) Luminescence decay (black scatter) for mer-[V(ddpd $\left.)_{2}\right]\left[\mathrm{PF}_{6}\right]_{3}$ in deoxygenated ${ }^{\mathrm{n}} \mathrm{PrCN}$ at $77 \mathrm{~K}$ ( $\lambda_{\mathrm{exc}}=306 \mathrm{~nm}, \lambda_{\mathrm{em}}=416 \mathrm{~nm}$ ) with biexponential fit function (red) and instrument response function (gray) and b) luminescence decay (black scatter) for mer- $\left[\mathrm{V}(\mathrm{ddpd})_{2}\right]\left[\mathrm{PF}_{6}\right]_{3}$ in deoxygenated $\mathrm{CD}_{3} \mathrm{CN}$ at $298 \mathrm{~K}$ ( $\lambda_{\text {exc }}=306 \mathrm{~nm}, \lambda_{\mathrm{em}}=390 \mathrm{~nm}$ ) with biexponential fit function (red) and instrument response function (gray). 
a)

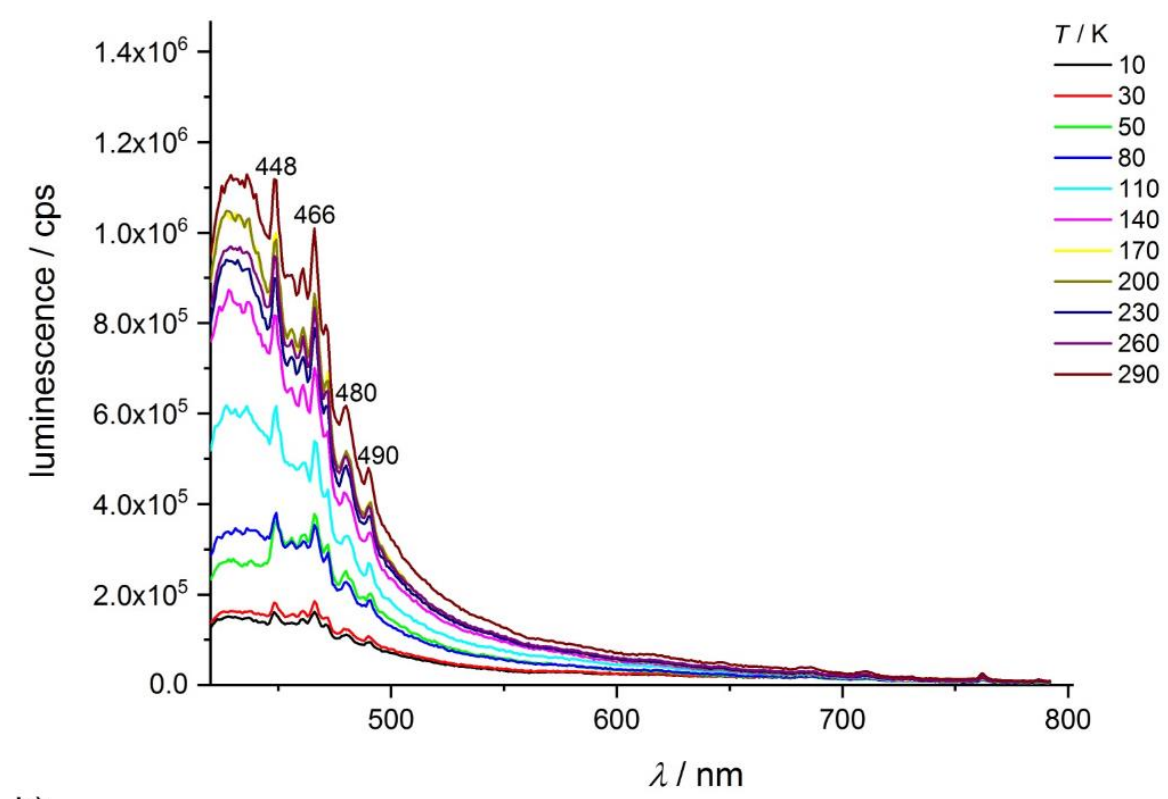

b)

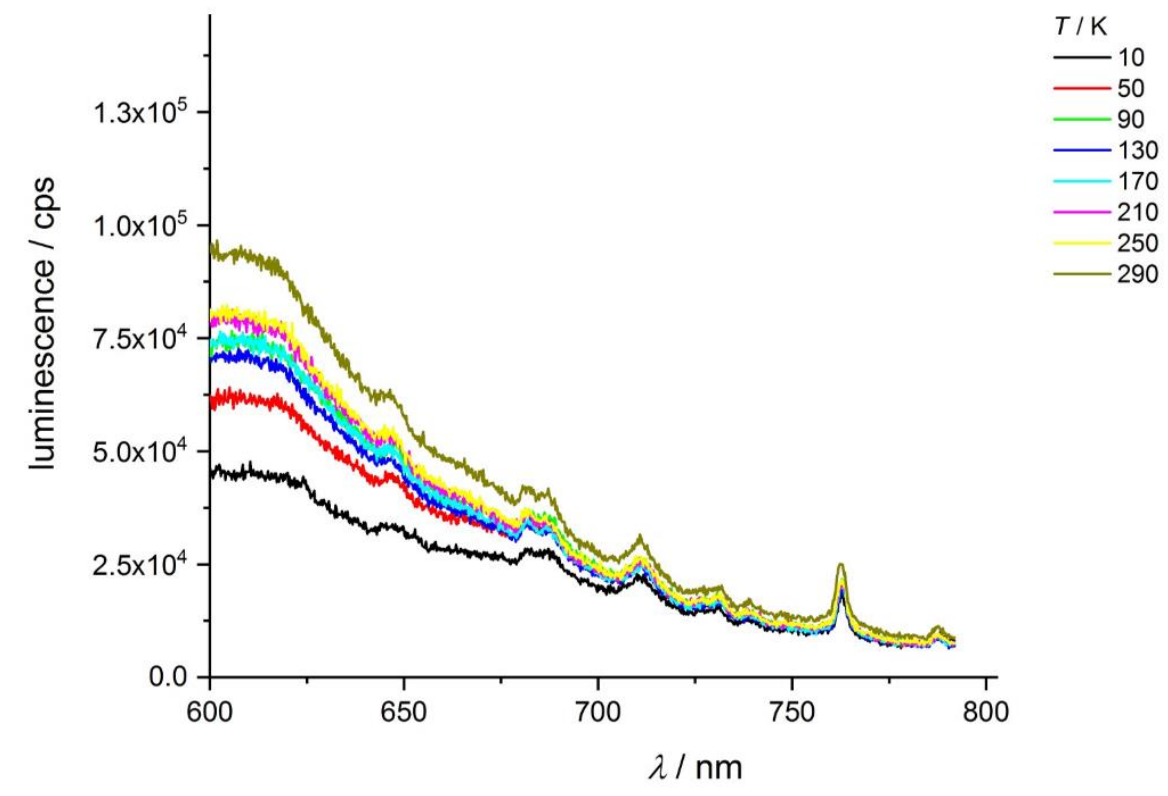

c)

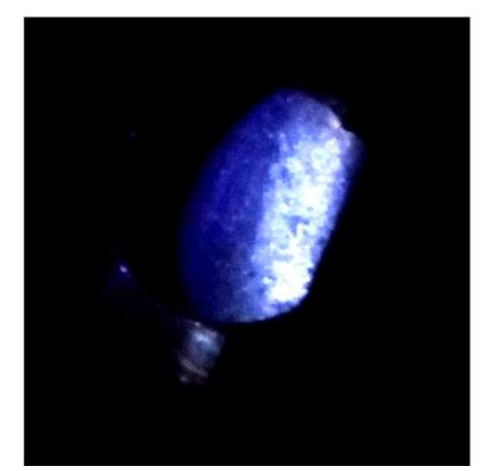

Figure S14. Emission spectra of mer- $\left[\mathrm{V}(\mathrm{ddpd})_{2}\right]\left[\mathrm{PF}_{6}\right]_{3}$ in a $\mathrm{KBr}$ pellet at different temperatures using a. 420 and b. $600 \mathrm{~nm}$ longpass filters; $\lambda_{\mathrm{exc}}=400 \mathrm{~nm}$. The quite surprising decrease of the intensity of blue fluorescence upon cooling the sample may be explained by a more favorable ISC to the singlet manifold at low temperature, compared to other radiative and non-radiative processes. c. Photograph of mer$\left[\mathrm{V}(\mathrm{ddpd})_{2}\right]\left[\mathrm{PF}_{6}\right]_{3}$ in a $\mathrm{KBr}$ pellet under excitation with $\lambda_{\text {exc }}=350 \mathrm{~nm}(290 \mathrm{~K})$. 


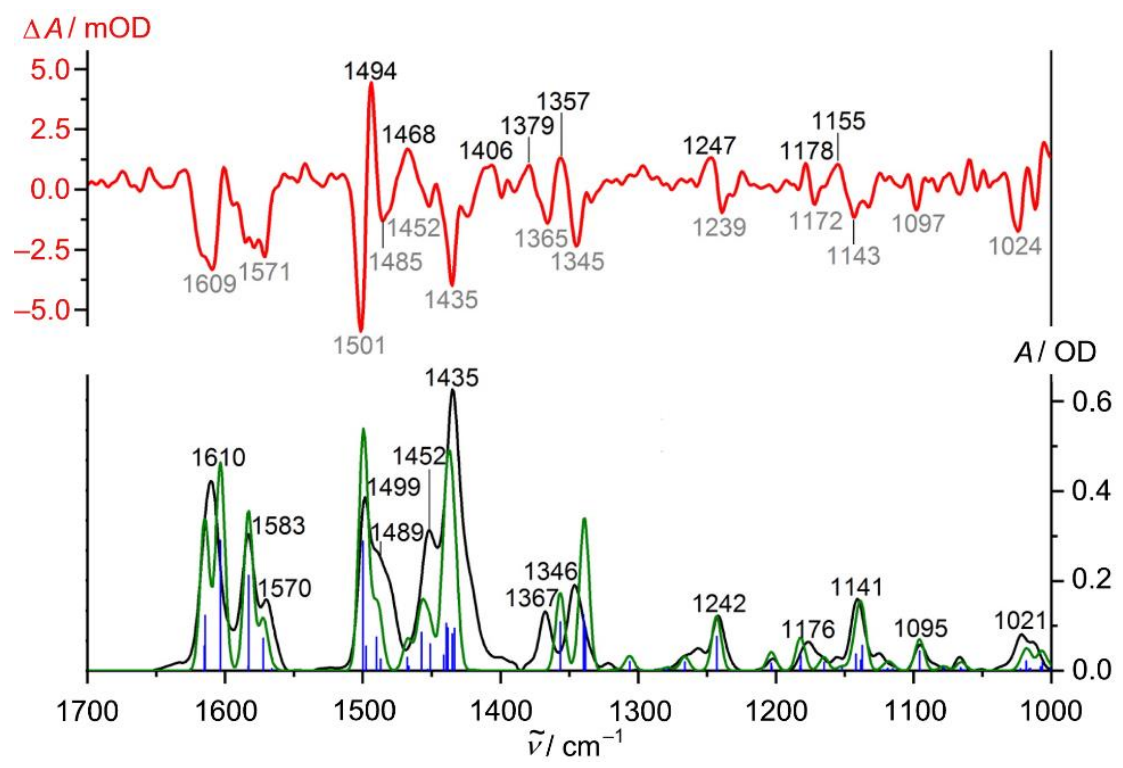

Figure S15. Step-scan FTIR spectrum of $\left[\mathrm{V}(\mathrm{ddpd})_{2}\right]\left[\mathrm{PF}_{6}\right]_{3}(\mathrm{KBr}$ pellet, $20 \mathrm{~K}) 0$ to $300 \mathrm{~ns}$ after laser excitation at $\lambda_{\text {exc }}=355 \mathrm{~nm}$ (red) and ground state FTIR spectrum (black) (KBr pellet, $\left.20 \mathrm{~K}\right)$, DFT-UKS calculated IR absorption transitions (blue) (scaled by 0.98), and DFT-UKS calculated IR spectrum (green) of [V(ddpd) $\left.{ }_{2}\right]^{3+}$ (scaled by 0.98 , $\mathrm{FWHM}=8 \mathrm{~cm}^{-1}$, gaussian profile).

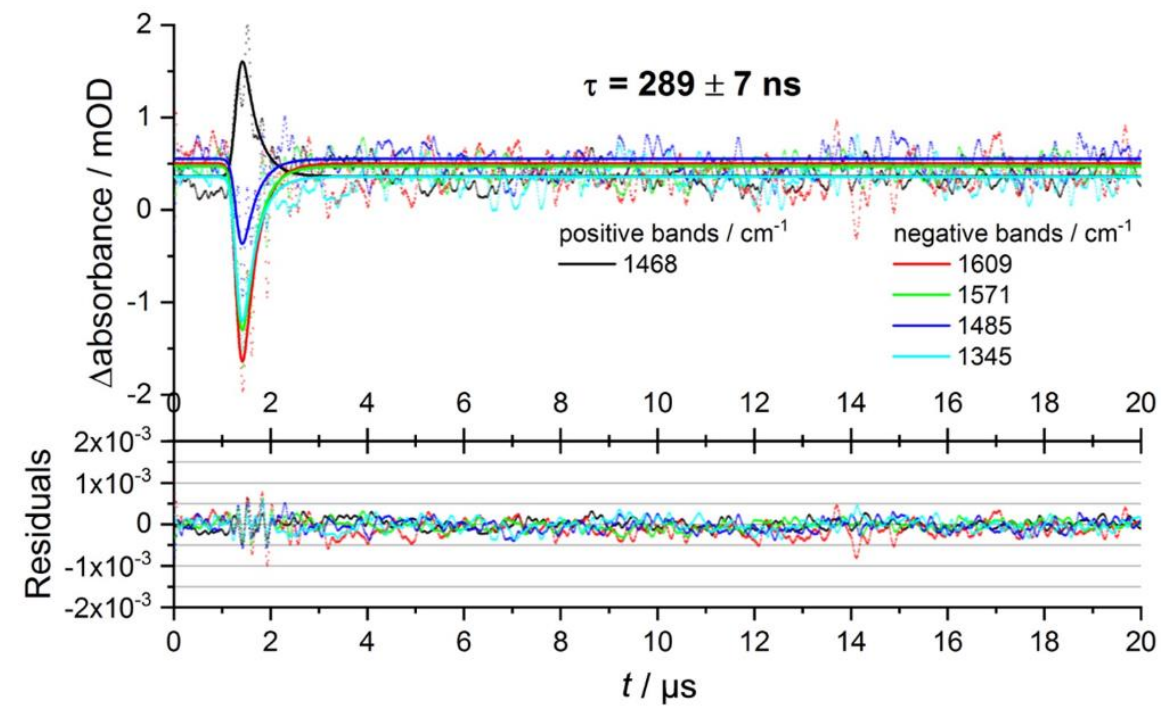

Figure S16. Global monoexponential fit and residuals performed for the most prominent positive and negative peaks in the step-scan spectrum of $\left[\mathrm{V}(\mathrm{ddpd})_{2}\right]\left[\mathrm{PF}_{6}\right]_{3}(\mathrm{KBr}$ pellet, $20 \mathrm{~K})$. 


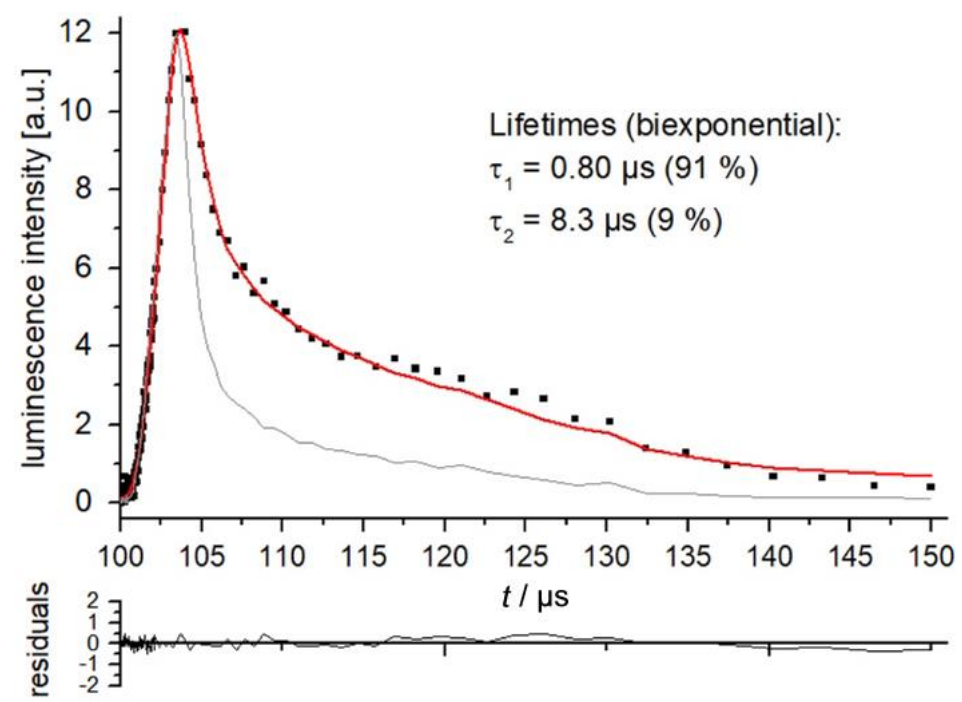

Figure S17. Luminescence decay (black scatter) for mer- $\left[\mathrm{V}\left(\left[\mathrm{D}_{17}\right]-\mathrm{ddpd}\right)_{2}\right]\left[\mathrm{PF} \mathrm{F}_{6}\right]_{3}$ in deoxygenated ${ }^{\mathrm{n} B u C N}$ at $77 \mathrm{~K}\left(\lambda_{\mathrm{exc}}=300 \mathrm{~nm}, \lambda_{\mathrm{em}}=1110 \mathrm{~nm}\right.$, emission path: long pass filter RG850) with biexponential fit function (red) and instrument response function (gray).

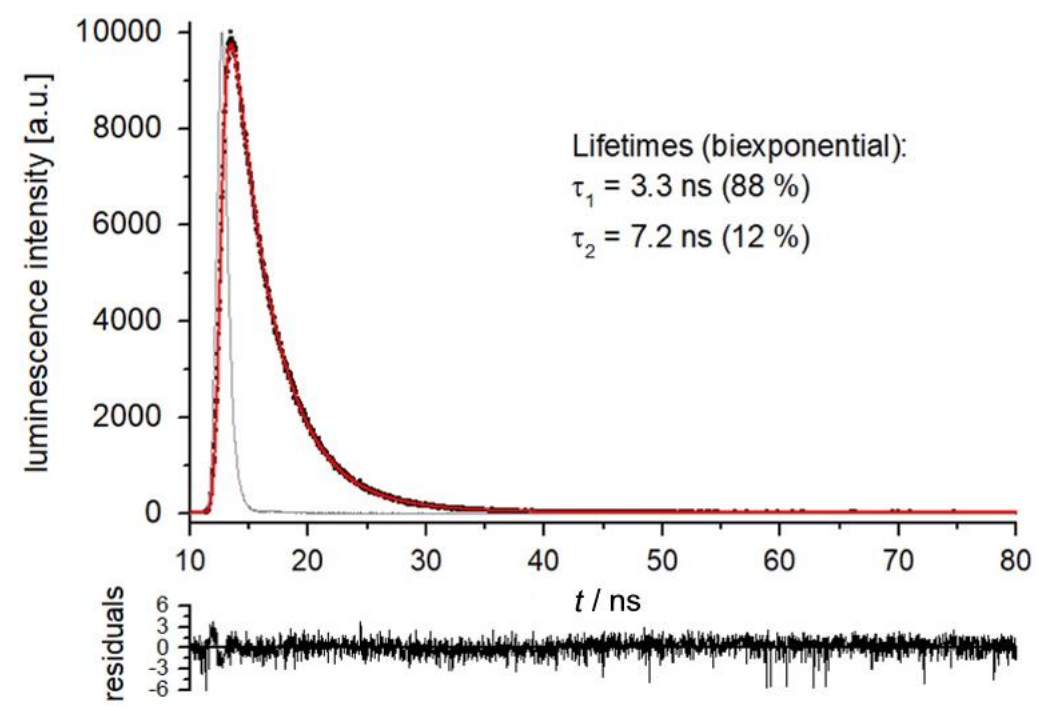

Figure S18. Luminescence decay (black scatter) for mer- $\left[\mathrm{V}\left(\left[\mathrm{D}_{17}\right]-\mathrm{ddpd}\right)_{2}\right]\left[\mathrm{PF}_{6}\right]_{3}$ in deoxygenated $\mathrm{CD}_{3} \mathrm{CN}$ at 298 $\mathrm{K}\left(\lambda_{\mathrm{exc}}=306 \mathrm{~nm}, \lambda_{\mathrm{em}}=390 \mathrm{~nm}\right)$ with biexponential fit function (red) and instrument response function (gray). 

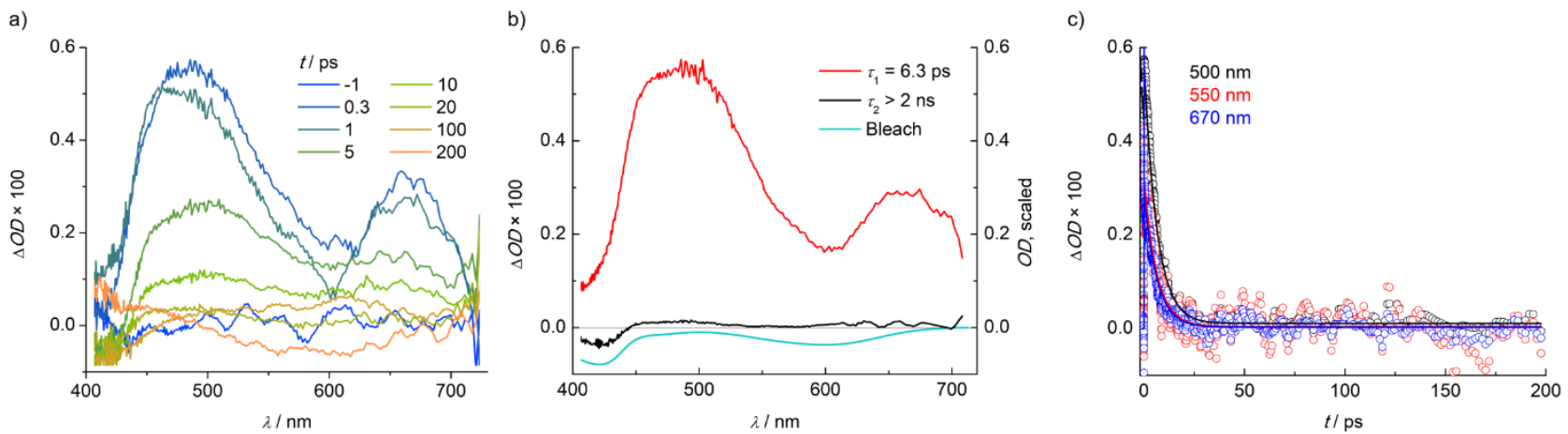

Figure S19. a. Transient absorption spectra of [ $\left.\mathrm{V}^{\prime \prime \prime}(\mathrm{ddpd})_{2}\right]\left[\mathrm{PF}_{6}\right]_{3}$ in $\mathrm{CH}_{3} \mathrm{CN}$ excited with $620 \mathrm{~nm}$ laser pulses. b. Decay associated amplitude spectra labelled by the corresponding time constants and compared to the bleach. c. Transient signals as a function of the pump-probe delay time observed at $500 \mathrm{~nm}$ (black), $550 \mathrm{~nm}$ (red) and $670 \mathrm{~nm}$ (blue).
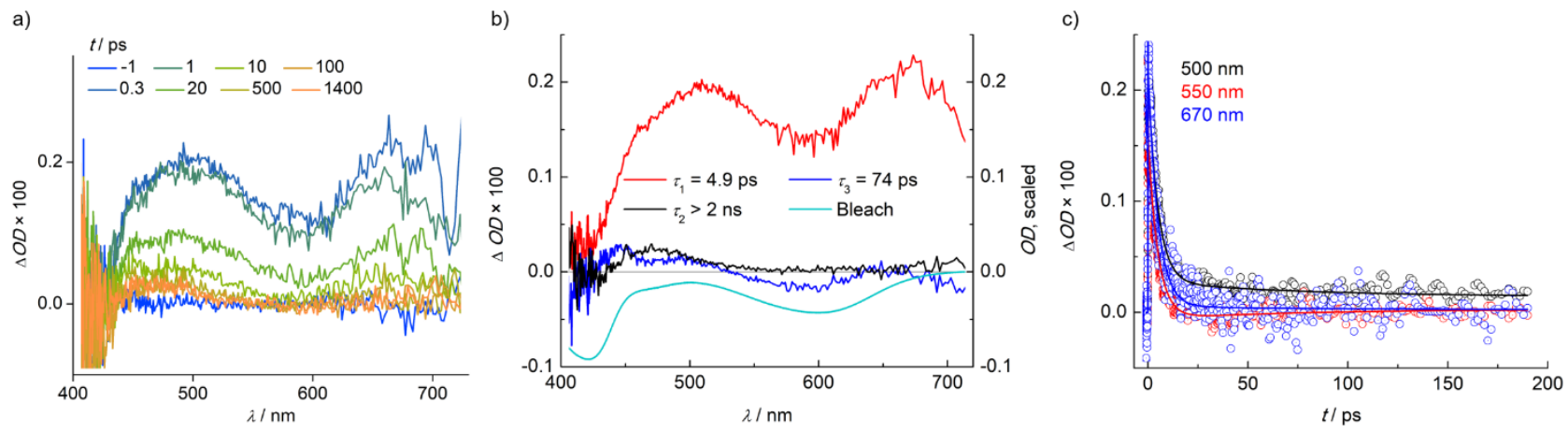

Figure S20. a. Transient absorption spectra of $\left[\mathrm{V}^{\prime \prime \prime}(\mathrm{ddpd})_{2}\right]\left[\mathrm{PF}_{6}\right]_{3}$ in $\mathrm{CH}_{3} \mathrm{CN}$ excited with $400 \mathrm{~nm}$ laser pulses. b. Decay associated amplitude spectra labelled by the corresponding time constants and compared to the bleach. c. Transient signals as a function of the pump-probe delay time observed at $500 \mathrm{~nm}$ (black), $550 \mathrm{~nm}$ (red) and $670 \mathrm{~nm}$ (blue). 
Table S8. TD-DFT-RKS relative energies $(\mathrm{eV})$ with respect to the triplet ground state ${ }^{3} \mathrm{~T}_{1 \mathrm{~g}}$ and corresponding assignment based on the largest contribution of the charge transfer numbers (Fig. S21) of the electronic states used to parameterize the potential energy surfaces employed in the SHARC-LVC simulations. Irreducible representations labels correspond to an idealized octahedral geometry (Fig. S1).

\begin{tabular}{llclc}
\hline State & Triplets & $\Delta E / \mathrm{eV}$ & Singlets & $\Delta E / \mathrm{eV}$ \\
\hline 1 & $1^{3} \mathrm{MC}\left({ }^{3} \mathrm{~T}_{1 \mathrm{~g}}\right)$ & 0 & $1^{1} \mathrm{MC}\left({ }^{1} \mathrm{E}_{\mathrm{g}}\right)$ & 1.16 \\
2 & $2^{3} \mathrm{MC}\left({ }^{3} \mathrm{~T}_{1 \mathrm{~g}}\right)$ & 0.26 & $2^{1} \mathrm{MC}\left({ }^{1} \mathrm{~T}_{2 \mathrm{~g}}\right)$ & 1.36 \\
3 & $3^{3} \mathrm{MC}\left({ }^{3} \mathrm{~T}_{2 \mathrm{~g}}\right)$ & 2.79 & $3^{1} \mathrm{MC}$ & 1.47 \\
4 & $4^{3} \mathrm{MC}\left({ }^{3} \mathrm{~T}_{2 \mathrm{~g}}\right)$ & 3.07 & $1^{1} \mathrm{LMCT}$ & 3.44 \\
5 & $1^{3} \mathrm{LMCT}$ & 3.33 & $2^{1} \mathrm{LMCT}$ & 3.50 \\
6 & $2^{3} \mathrm{LMCT}$ & 3.39 & $3^{1} \mathrm{LMCT}$ & 3.59 \\
7 & $3^{3} \mathrm{LMCT}$ & 3.42 & $4^{1} \mathrm{LMCT}$ & 3.64 \\
8 & $4^{3} \mathrm{LMCT}$ & 3.48 & $4^{1} \mathrm{MC}$ & 3.72 \\
9 & $5^{3} \mathrm{LMCT}$ & 3.78 & $5^{1} \mathrm{MC}$ & 3.79 \\
10 & $6^{3} \mathrm{LMCT}$ & 3.79 & $5^{1} \mathrm{LMCT}$ & 3.94 \\
11 & $7^{3} \mathrm{LMCT}$ & 3.90 & $6^{1} \mathrm{LMCT}$ & 3.96 \\
12 & $8^{3} \mathrm{LMCT}$ & 3.92 & $7^{1} \mathrm{LMCT}$ & 4.08 \\
\hline
\end{tabular}

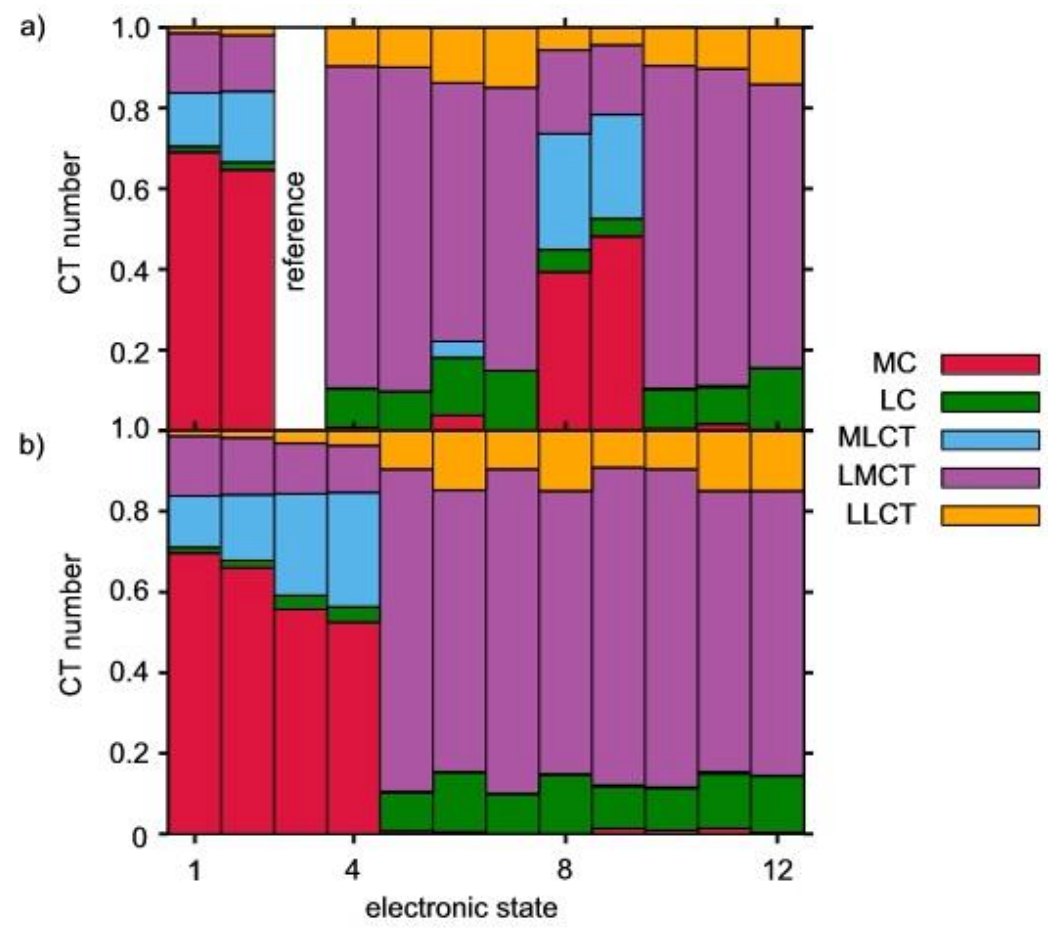

Figure S21. TD-DFT-RKS charge transfer (CT) numbers defined from 0 to 1 of the lowest-lying 12 singlets (a) and 12 triplets (b) states, calculated with respect to the closed-shell reference $3^{1} \mathrm{MC}\left({ }^{1} \mathrm{~A}_{1 \mathrm{~g}}\right)$. The reference state is $100 \%$ closed-shell and serves as a reference for the CT numbers calculated. 


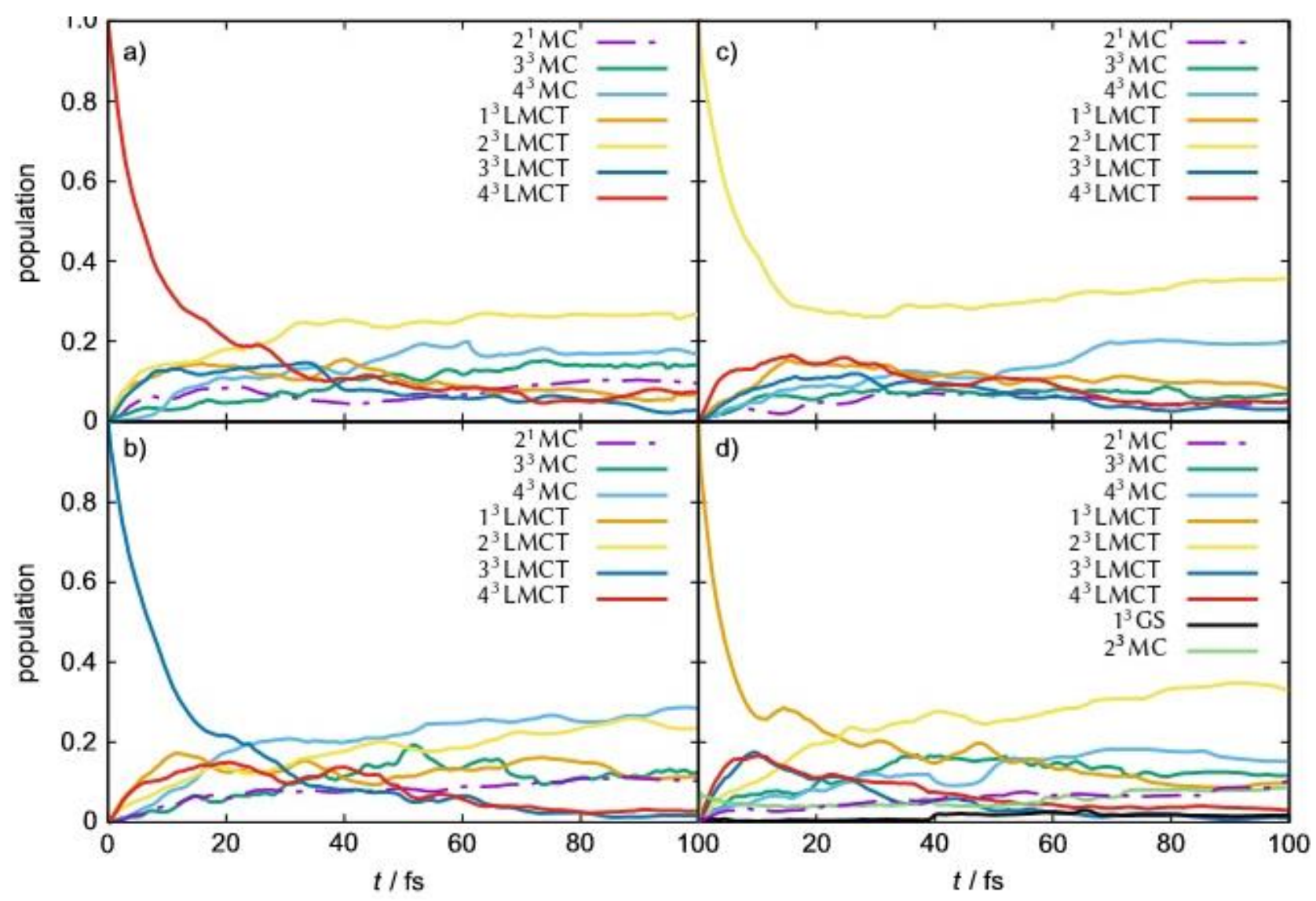

Figure S22. Time-evolution of the population on the excited states during the first $100 \mathrm{fs}$, after starting the dynamics on a) $4^{3} \mathrm{LMCT}$, b) $3^{3} \mathrm{LMCT}$, c) $2^{3} \mathrm{LMCT}$, d) $1^{3} \mathrm{LMCT}$ triplet states. Population in any other singlet state other than $2^{1} \mathrm{MC}$ is negligible and not plotted. 
Table S9. Cartesian coordinates of the minimum energy geometry of the $2^{1} \mathrm{MC}$ singlet state, optimized within the LVC method.

$-0.66530157968787$ $-0.33031990692470$ 0.41830831745261 0.97362870509963 1.61181540473234 $-0.72354413669044$ $-1.96074838838531$ $-3.22330616437540$ $-2.21011674159465$ $-1.11064559695091$ 0.58976681646651 $-0.51902350304275$ $-0.99699684623169$ $-0.16320443140994$ $-0.35556178466572$ 0.51176234304264 0.89902833180966 0.75868681650198 1. 34566669070994 0.27580951908176 0.42018420640678 $-0.39584601781559$ 1. 37894137222654 0.26254884577769 1. 15439748411093 2.10641939261553 2.25574519220159 2. 97403726640090 3. 76278574276453 2.86302853677891 3.49707496006560 1.81763156898550 2.83029928965059 2.41554656988593 3. 5508733370355 3. 42879715613805 0.44216636160675 0.46573486730863 1.41703906901873 $-0.73262762177129$ $-0.74238450534174$ $-1.92899150285652$ $-2.87962140492781$ $-1.89273795903647$ $-2.81901711234949$ $-1.67220076396436$ $-0.98933712529239$ $-2.15687315540798$ $-1.86512504601290$ $-3.01035880731720$ $-3.40025978996045$ $-3.38350807185125$ $-4.08062037759673$ $-2.86067965063365$ $-3.70493630437212$ $-3.04190831110575$ $-4.75507503727469$ $-3.58814105987102$ $-3.17180039490112$ $-4.11306109280554$ $-4.91248388501292$ $-3.97406588955119$ $-4.65613641102556$ $-2.98017046603333$ $-2.83517191274696$ $-2.12851454730312$ $-1.45842223194776$ $-0.81466042114834$ $-1.27972973059534$ $-2.56663951869531$ 0.22495910042885 1.12055930549567 0.81802530346211 1. 83052754324382 2. 38955024184869 2.75514231091441 2.07673153270343 3. 73629512792687 3.08593957658572
4.73327191559267 4.49554366729562 2.21830701812423 3.50540643057365 5.06453779574661

4.76766598674381 3. 16994764007497 4.11306267448654 5.95501601848848

7.76960212804624 6.42517020550181

5.56679860144001 6.48213429825880 5.50958067229945 6. 39299189250326 4. 31800507016179 4. 22851529398475 3.25593348824104 2. 36340268708133 3. 34952219944841 0.85020353375555 0.77755344403761 0.67430009465801 0.07566127797111 2. 36476309861683 1. 42356718411031 0.51061526752509 1.76629990326041 1.08027705090921 2.99025708861967 3. 31985076379143 3. 82320791161327 5.97887585408924 6.99646409452291 5.67230931017101 5.96338504169185 5.38973471048780 6.34793466226565 6. 78735923738471

6.65128543688819 7. 40302113893741 5.92906992859351 6.11776359400517 5. 00037101855265 4.50676085023559 2. 11464319120735 2. 32687239570611

0.84205131370184

0.01176335787152 0.65100071089963 $-0.34014703517561$ 1.73340937704325 1.60134843901535 3. 01531348345467

3. 82438704648768 3.05752658744568 3. 49933672866476 4.72646958234386 5.50367725461328 6.48231054318854 6.10384131769645 8.00933775827573

8.81979055084680

8.45680858404359

9.63489712391607

7. 37398690254809

8.99550642510190

8.96943559455970

9.94759527925353

8.97082259954718 7. 63515938090614 8.71230282173515 9. 67679693582683 6.25881473429048 8.53412485803401 7.28051998723144 5.26299380217903 7.09459911151002 9. 36744507524232
1. 15414806626787 3. 24011128941319 2. 87578640975785 0.94630448773096 $-0.70547438832152$ $-0.90727949908829$

1.08780284944625

2.85863757136509

1.67409350682190

0.59882839517929

1. 45105292787956

4.08464141971885

3. 63165367747580

5.48352260559947

6.10453377228770 6.06408638013690 7. 15602764374424 5.19995115897545 5.61351377319930 3.77455144119524 3.57052585170090

4. 32684050305551 4.11198294918403 2.76831582681121 1.67101644509280 1. 25941935733429 1. 81405784265292 0.23081403718095 $-0.05494221860542$ $-0.42628638110484$ $-1.23583990510299$ $-0.07354996768462$ $-0.90011021511064$ $-0.96780825332937$ $-1.75596654545864$ 0.08606352508797 $-1.36280251430980$ $-2.45814903615440$ $-2.81081756219200$ $-3.09104037879572$ $-3.93830763666378$ $-2.67105828474415$ $-3.16034885436779$ $-1.58049928373020$

$-1.17595517763402$

0.26628263347842 $-0.54156214987688$

0.48371391037265

$-0.15931420609855$

1.57491682377841

1.79390915664606

2. 36839128304000

3. 18276761788482

2.07944393277739

4. 23523611756562

4. 66273815789241

4.27620673752258

4.82478436598885

2. 53685243927383

3. 21399326932164

3. 83079600124229

3.19294090222228

3. 86077222364849

2. 32222283020250

2. 30759569178623

1. 52735339577138

$-0.17258183971986$

$-1.08741231606597$

0.44055814387018

$-0.49474886031302$ 0.99965310256870 0.98126581737906 0.60260621904721 1.95615033551378 1. 50832530143104 2.00643290806222 2.30093049901599 2.42034138169754 1. 54281956117543 


\section{References.}

1. Breivogel, A.; Förster, C.; Heinze, K. A Heteroleptic Bis(tridentate)ruthenium(II) Polypyridine Complex with Improved Photophysical Properties and Integrated Functionalizability. Inorg. Chem. 2010, 49, 70527056.

2. Anderson, S. J.; Wells, F. J.; Wilkinson, G.; Hussain, B.; Hursthouse, M. B. 1,2Bis(dimethyl)phosphinoethane complexes of molybdenum and vanadium. X-ray crystal structure of trans-[MoCl$\left.\left(\eta^{2}-\mathrm{NCMe}\right)(\mathrm{dmpe})_{2}\right] \mathrm{BPh}_{4}, \quad$ trans- $\left[(\mathrm{SPh})_{2}(\mathrm{dmpe})_{2}\right], \quad$ trans- $\left[\mathrm{V}(\mathrm{NCMe})_{2}(\mathrm{dmpe})_{2}\right] \mathrm{BPh}_{4}$, trans$\left[\mathrm{V}\left(\mathrm{CNBu}^{\mathrm{t}}\right)_{2}(\mathrm{dmpe})_{2}\right]\left(\mathrm{PF}_{6}\right)_{2}$. Polyhedron 1988, 7, 2615-2626.

3. Fulmer, G. R.; Miller, A. J. M.; Sherden, N. H.; Gottlieb, H. E.; Nudelman, A.; Stoltz, B. M.; Bercaw, J. E.; Goldberg, K. I. NMR Chemical Shifts of Trace Impurities: Common Laboratory Solvents, Organics, and Gases in Deuterated Solvents Relevant to the Organometallic Chemist. Organometallics 2010, 29, 21762179.

4. Melhuish, W. H. Quantum efficiencies of fluorescence of organic substrates: effect of solvent and concentration of the fluorescent solute. J. Phys. Chem. 1961, 65, 229-235.

5. Meshkova, S. B.; Topilova, Z. M.; Bolshoy, Z. M.; Beltyukova, S. V.; Tsvirko, M. P.; Venchikov, V. Y. Quantum Efficiency of the Luminescence of Ytterbium(III) $\beta$-Diketonates. Acta Phys. Pol. A 1999, 95, 983990.

6. a) Zimmer, M.; Rupp, F.; Singer, P.; Walz, F.; Breher, F.; Klopper, W.; Diller, R.; Gerhards, M. Time-resolved IR spectroscopy of a trinuclear palladium complex in solution. Phys. Chem. Chem. Phys. 2015, 17, 1413814144; b) Bäppler, F.; Zimmer, M.; Dietrich, F.; Gruppe, M.; Wallesch, M.; Volz, D.; Bräse, S.; Gerhards, M.; Diller, R. Photophysical dynamics of a binuclear $\mathrm{Cu}(\mathrm{I})$-emitter on the fs to $\mu$ s timescale, in solid phase and in solution. Phys. Chem. Chem. Phys. 2017, 19, 29438-29448; c) Zimmer, M.; Dietrich, F.; Volz, D.; Bräse, S.; Gerhards, M. Solid-State Step-Scan FTIR Spectroscopy of Binuclear Copper(I) Complexes. ChemPhysChem 2017, 18, 3023-3029.

7. STOE \& Cie, X-Red, STOE \& Cie, Darmstadt, Germany 2002.

8. Blessing, R. H. An empirical correction for absorption anisotropy. Acta Crystallogr. Sect. A 1995, 51, 3338.

9. Spek, A. L. Structure validation in chemical crystallography. Acta Crystallogr. Sect. D 2009, 65, 148-55.

10. Sheldrick, G. M. SHELXL-2014/7, University of Göttingen, Göttingen, Germany, 2014.

11. Sheldrick, G. M. SHELXT - Integrated space-group and crystal-structure determination. Acta Crystallogr. Sect. A 2015, 71, 3-8.

12. Neese, F. The ORCA program system. WIREs Comput. Mol. Sci. 2012, 2, 73-78.

13. Becke, A. D. Density-functional thermochemistry. III. The role of exact exchange. J. Chem. Phys. 1993, 98, 5648-5652.

14. Weigend, F.; Ahlrichs, R. Balanced basis sets of split valence, triple zeta valence and quadruple zeta valence quality for $\mathrm{H}$ to Rn: Design and assessment of accuracy. Phys. Chem. Chem. Phys. 2005, 7, 32973305.

15. Weigend, F. Accurate Coulomb-fitting basis sets for H to Rn. Phys. Chem. Chem. Phys. 2006, 8, 10571065.

16. Neese, F.; Wennmohs, F.; Hansen, A.; Becker, U. Efficient, approximate and parallel Hartree-Fock and hybrid DFT calculations. A 'chain-of-spheres' algorithm for the Hartree-Fock exchange. Chem. Phys. 2009, 356, 98-109.

17. Izsák, R.; Neese, F. An overlap fitted chain of spheres exchange method. J. Chem. Phys. 2011, 135, 144105.

18. Pantazis, D. A.; Chen, X.-Y.; Landis, C. R.; Neese, F. All-Electron Scalar Relativistic Basis Sets for Third-Row Transition Metal Atoms. J. Chem. Theory Comput. 2008, 4, 908-919.

19. van Lenthe, E.; Baerends, E. J.; Snijders, J. G. Relativistic regular two-component Hamiltonians. J. Chem. Phys. 1993, 99, 4597-4610.

20. Grimme, S.; Antony, J.; Ehrlich, S.; Krieg, H. A consistent and accurate ab initio parametrization of density functional dispersion correction (DFT-D) for the 94 elements H-Pu. J. Chem. Phys. 2010, 132, 154104. 
21. Grimme, S.; Ehrlich, S.; Goerigk, L. Effect of the damping function in dispersion corrected density functional theory. J. Comput. Chem. 2011, 32, 1456-1465.

22. Barone, V.; Cossi, M. Quantum Calculation of Molecular Energies and Energy Gradients in Solution by a Conductor Solvent Model. J. Phys. Chem. A 1998, 102, 1995-2001.

23. Roos, B. O.; Taylor, P. R.; Siegbahn, P. E. M. A Complete Active Space SCF Method (CASSCF) Using a Density-Matrix Formulated Super-Cl Approach. Chem. Phys. 1980, 48, 157-173.

24. Siegbahn, P. E. M.; Almlof, J., Heiberg, A.; Roos, B. O. The Complete Active Space Scf (CASSCF) Method in a Newton-Raphson Formulation with Application to the HNO Molecule. J. Chem. Phys. 1981, 74, 2384-2396.

25. Angeli, C.; Cimiraglia, R.; Evangelisti, S.; Leininger, T.; Malrieu, J.-P. Introduction of n-electron valence states for multireference perturbation theory. J. Chem. Phys. 2001, 114, 10252-10264.

26. Angeli, C.; Cimiraglia, R. Multireference perturbation configuration interaction V. Third-order energy contributions in the Møller-Plesset and Epstein-Nesbet partitions. Theor. Chem. Acc. 2002, 107, 313-317.

27. Neese, F. Efficient and accurate approximations to the molecular spin-orbit coupling operator and their use in molecular g-tensor calculations. J. Chem. Phys. 2005, 122, 34107.

28. Hess, B. A.; Marian, C. M. In Computational Molecular Spectroscopy; Jensen, P. B., Ed.; Wiley: New York, 2000; p. 169ff.

29. Cahier, B.; Maurice, R.; Bolvin, H.; Mallah, T.; Guihéry, N. Tools for Predicting the Nature and Magnitude of Magnetic Anisotropy in Transition Metal Complexes: Application to $\mathrm{Co}(\mathrm{II})$ Complexes. Magnetochemistry 2016, 2, 31-45.

30. Roos, B. O.; Malmqvist, P.-Å. Relativistic quantum chemistry: the multiconfigurational approach. Phys. Chem. Chem. Phys. 2004, 6, 2919-2927.

31. Durand, P.; Malrieu, J.-P. In Advances in Chemical Physics: Ab Initio Methods in Quantum Chemistry; Lawley, K. P., Ed.; John Wiley \& Sons Ltd.: Hoboken, NJ, 1987; Vol. 67, Part 1, pp 321-412.

32. Pierloot, K. Transition metals compounds: Outstanding challenges for multiconfigurational methods. Int. J. Quantum Chem. 2011, 111, 3291-3301.

33. Richter, M.; Marquetand, P.; González-Vazquez, J.; Sola, I.; González, L. SHARC: ab Initio Molecular Dynamics with Surface Hopping in the Adiabatic Representation Including Arbitrary Couplings. J. Chem. Theory Comput. 2011, 7, 1253-1258.

34. Mai, S.; Marquetand, P.; González, L. WIREs Comput. Mol. Sci. 2018, e1370.

35. Mai, S.; Marquetand, P.; González, L. Int. J. Quantum Chem. 2015, 115, 1215-1231.

36. Mai, S.; Richter, M.; Heindl, M.; Menger, M. F. S. J.; Atkins, A.; Ruckenbauer, M.; Plasser, F.; Oppel, M.; Marquetand, P.; González, L. Sharc2.0: Surface Hopping Including Arbitrary Couplings - Program Package for Non-Adiabatic Dynamics (2018), available from http://sharc-md.org.

37. Plasser, F.; Gómez, S.; Menger, M. F. S. J.; Mai, S.; González, L. Highly efficient surface hopping dynamics using a linear vibronic coupling model. Phys. Chem. Chem. Phys. 2019, 21, 57-69.

38. a) Mai, S.; Plasser, F.; Dorn, J.; Fumana, M.; Daniel, C.; González, L. Quantitative wave function analysis for excited states of transition metal complexes. Coord. Chem. Rev. 2018, 361, 74-97; b) http://theodoreqc.sourceforge.net.

39. Granucci, G.; Persico, M. Direct semiclassical simulation of photochemical processes with semiempirical wave functions. J. Chem. Phys. 2001, 114, 10608.

40. Granucci, G.; Persico, M. Critical appraisal of the fewest switches algorithm for surface hopping. J. Chem. Phys. 2007, 126, 134114.

41. Alemany, P.; Casanova, D.; Alvarez, S.; Dryzun, C.; Avnir, D. Continuous Symmetry Measures: A New Tool in Quantum Chemistry. In Reviews in Computational Chemistry. Parrill, A. L., Lipkowitz, K. B., Eds., John Wiley \& Sons: Hoboken, NJ, USA, Volume 30, 2017, pp. 289-352.

42. Tanabe, Y.; Sugano, S. On the Absorption Spectra of Complex Ions. II. J. Phys. Soc. Jpn. 1954, 9, 766-779. 
43. Krzystek, J.; Ozarowski, A.; Telser, J.; Crans, D. C. High-frequency and -field electron paramagnetic resonance of vanadium(IV, III, and II) complexes. Coord. Chem. Rev. 2015, 301-302, 123-133.

44. Kittilstved, K. R.; Sorgho, L. A.; Amstutz, N.; Tregenna-Piggott, P. L. W.; Hauser, A. Ground-State Electronic Structure of Vanadium(III) Trisoxalate in Hydrated Compounds. Inorg. Chem. 2009, 48, 7750-7764.

45. Jung, J.; Jung, Y.; Park, O. Preparation of 2-chloropyridine. Synth. Commun. 2001, 31, 2507-2511.

46. Jha, A. K.; Jain, N. The microwave-assisted ortho-alkylation of azine N-oxides with N-tosylhydrazones catalyzed by copper(I) iodide. Chem. Commun. 2016, 52, 1831-1834.

47. Esaki, H.; Ito, N.; Sakai, S.; Maegawa, T.; Monguchia, Y.; Sajikia, H. General method of obtaining deuterium-labeled heterocyclic compounds using neutral $\mathrm{D}_{2} \mathrm{O}$ with heterogeneous $\mathrm{Pd} / \mathrm{C}$. Tetrahedron 2006, 62, 10954-10961.

48. Dirvanauskas, A.; Galavotti, R.; Lunghi, A.; Nicolini, A.; Roncaglia, F.; Totti, F.; Cornia, A. Solution structure of a pentachromium(II) single molecule magnet from DFT calculations, isotopic labelling and multinuclear NMR spectroscopy. Dalton Trans. 2018, 47, 585-595.

49. Wang, C.; Otto, S.; Dorn, M.; Kreidt, E.; Lebon, J.; Sršan, L.; Di Martino-Fumo, P.; Gerhards, M.; ReschGenger, U.; Seitz, M.; Heinze, K. Deuterated Molecular Ruby with Record Luminescence Quantum Yield. Angew. Chem. Int. Ed. 2018, 57, 1112-1116. 